\title{
Assessment of groundwater quality for drinking and irrigation purposes in arid areas of Rajasthan, India
}

\author{
Veena Chaudhary ${ }^{1} \cdot$ S. Satheeshkumar ${ }^{2}$
}

Received: 10 December 2016 / Accepted: 1 November 2018 / Published online: 8 November 2018

(c) The Author(s) 2018

\begin{abstract}
The quality of groundwater is poorly understood in the arid northwest part of Rajasthan, whereas it is the only source of drinking and irrigation and the residents consume it without any prior treatment. This study illustrates the qualitative analysis of groundwater and its suitability in the bulk samples collected from three different canal catchment areas. Most of the samples were identified for higher values of EC, TDS, TH and fluoride, therefore considered posing restriction to drinking use. The abundance of major ions was found in the order of $\mathrm{Na}^{+}>\mathrm{Ca}^{+2}>\mathrm{Mg}^{+2}>\mathrm{K}^{+}=\mathrm{Cl}^{-}>\mathrm{HCO}_{3}^{-}>\mathrm{SO}_{4}^{-2}>\mathrm{NO}_{3}^{-}>\mathrm{F}^{-}$. The irrigation quality parameters such as sodium adsorption ratio, $\% \mathrm{Na}$, residual sodium carbonate, residual sodium bicarbonate, Kelley's index, potential salinity, magnesium hazard, $\mathrm{Mg} / \mathrm{Ca}$ ratio and permeability index were calculated and discussed thoroughly in combination with Wilcox, USSL and Doneen diagrams. Most of the samples belong to predominant $\mathrm{Cl}^{-}$and $\mathrm{Na}^{+}$in hydrogeochemical studies. According to USSL diagram, majority of the samples fall under $\mathrm{C}_{4} \mathrm{~S}_{1}$ class. Furthermore, groundwater chemistry was found mainly influenced by evaporation-crystallization in Gibbs variation diagram. This study suggested that groundwater is unsafe for drinking purpose without purification and quality measures should be considered while cropping in its irrigation use.
\end{abstract}

Keywords Groundwater quality $\cdot$ Canal catchment area $\cdot$ Drinking $\cdot$ Irrigation

\section{Introduction}

Groundwater is a life-sustaining resource that plays a central part in irrigated agriculture and influences the health of many ecosystems. But unsustainable depletion of groundwater has been documented on both regional and global scales (Rodell et al. 2009; Gleeson et al. 2012; MacDonald et al. 2016). The quality of groundwater is equally important to its quantity owing to the suitability of water for various purposes (Kumar et al. 2009; Subramani et al. 2005). In recent years, intensive agricultural activities, domestic and industrial discharge, over-exploitation, uneven rainfall and mismanagement of groundwater have raised serious concern regarding groundwater contamination (Jain et al. 2010; Salifu et al. 2013; Fianko et al. 2010). The World Health

Veena Chaudhary

veena_chaudhary@yahoo.co.in

1 Department of Chemistry, C.S.S.S. PG College, Machhra, Meerut, UP, India

2 Department of Geology, Periyar University, Salem, Tamil Nadu 636011, India
Organization reports that every year more than 3.4 million people die as a result of water-related diseases and a leading cause of death around the world.

Agriculture is demographically the broadcast economic sector and plays a significant role in the overall socioeconomic growth of India. The agriculture growth rate in India GDP has been growing earlier but in the last few years it is constantly declining as 5.0, 1.7, 3.8 and 1.1 in 1996-2000, 2001-2005, 2006-2010 and 2011-2015, respectively. The improvements in irrigation, infrastructure, quality seeds, innovative mechanization, chemical fertilizers and pesticides have helped India to make it seventh largest agricultural exporter worldwide. But, on the other side, massive use of chemical fertilizers and pesticides deteriorated both groundwater quality and soil health (Arumugam and Elangovan 2009; Kurdi et al. 2013; Rajmohan and Elango 2005).

Suitability of groundwater for domestic and irrigation purposes is determined by its groundwater geochemistry because each groundwater system has a unique chemical composition and any alteration depends on several factors such as rock-water interaction, mineral dissolution, soil-water interaction, interaction time, temperature and

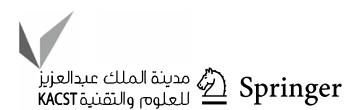


anthropogenic activities (Back and Hanshaw 1965; Drever 1988; Stallard and Edmond 1983; Subba Rao et al. 2002). In order to prevent water-borne diseases, damage to sensitive crops and soil health, groundwater quality measures should be ensured (Sarkar and Hassan 2006; Bauder et al. 2004; Little et al. 2010).

The northwest part of Rajasthan state in India has been selected for the present study. Although three canal systems (Indira Gandhi (IGNP), Bhakra and Gang Canals) are available in the region, an insufficient water supply of canal water and low rainfall made localities depend on the groundwater. Agriculture and livestock are the most common economic activities in the study area; therefore, a hydrogeochemical investigation was carried out to identify groundwater chemistry and its suitability for irrigation and drinking purposes.

\section{Study area}

The study area is comprised of northwest part of Rajasthan, located between $28.4^{\circ}-30.3^{\circ}$ north latitude and $72.3^{\circ}-75.3^{\circ}$ east longitude and at an altitude of $175.6 \mathrm{~m}$ above mean sea level. To assess groundwater resources and the possibilities of their utilization, this part has been classified under arid climatic region. Rainfall is the main source of annual replenishing groundwater resource which is scanty and mostly up to $0.025 \mathrm{~m}$ in the region. The general trend of the slope in the region is from the northeast to southwest. The presence and availability of groundwater vary greatly with changes in topography, subsurface geology and the prevailing climate in the region.

Geology of the region is marked by a thick cover of blown sand and alluvium except for a few isolated patches of recent calcareous and sandy sediments associated with gypsum. The oldest rocks in the area belong to the Aravalli subgroup which includes phyllite, shale and quartz veins. These are overlain by the rocks of upper Vindhyans which are entirely made up of bright to pale red, fine- and medium-grained compact sand stone and silt stone. The windblown sand mainly consists of quartz with minor biotite and magnetite. Gypsum-rich beds are found in shallow depression surrounded by sand dunes. Apart from this, scattered occurrences of saltpeter are seen in number of intradunal basins. The northern part of the area is characterized by arid soils which are light yellowish brown to pale in color. The presence of calcareous concretions has been noticed in the depth range of $75-100 \mathrm{~cm}$. Soils are deep and moderately drained. Permeability is moderate to moderately slow, and water holding capacity and natural fertility are generally poor. Loamy sand and sandy loam are the predominant types of soil met within this soil group. At places, patches of sand also occur. The southern portion of the region is characterized by desert soils, which are very pale brown to yellowish brown in color and are generally devoid of lime concretions. Soils are generally well drained to excessively drained and have low moisture holding capacity and high permeability. The principal aquifer in the region is alluvium comprising sand, silt, clay and gravel. Groundwater occurs under both water table and confined conditions. The thickness of shallow water table aquifer varies from a few meters to about $80 \mathrm{~m}$. Confined aquifer is found both in alluvium and under lying sandstone and depth ranging from 90 to $100 \mathrm{~m}$.

Porosity, hydraulic conductivity and transmissivity are some important physical concepts to understand the degree to which a body of rock or sediment will function as a groundwater resource. Porosity does not tell the entire story about the availability of groundwater in the subsurface. The pore spaces must also interconnect and be large enough so that water can move through the ground to be extracted from a well or discharged to a water body. The term 'effective porosity' refers to the degree of interconnectedness of pore spaces. For coarse sediments, such as the sand and gravel encountered in alluvial groundwater basins, the effective porosity is often nearly equal to the overall porosity. Specific yield is the fractional amount of water that would drain freely from rocks or sediments due to gravity and describes the portion of the groundwater that could actually be available for extraction. Lower porosity in the soil of study area makes excellent sources of groundwater because of the high specific yield, which allows the groundwater to flow to wells.

Another major property related to understanding water movement in the subsurface is hydraulic conductivity. Hydraulic conductivity is a measure of a rock or sediment's ability to transmit water and often used interchangeably with the term permeability. The size, shape and interconnectedness of pore spaces affect hydraulic conductivity. Hydraulic conductivity is usually expressed in units of length/time: feet/day, meters/day or gallons/ day/square foot. Hydraulic conductivity values in rocks range over many orders of magnitude from a low-permeability unfractured crystalline rock at about $10^{-8}$ feet/day to a highly permeable well-sorted gravel at greater than $10^{4} \mathrm{feet} /$ day.

Transmissivity is a measure of the aquifer's ability to transmit groundwater through its entire saturated thickness and relates closely to the potential yield of wells. Transmissivity is defined as the product of the hydraulic conductivity and the saturated thickness of the aquifer. The value of transmissivity in the study area is $375-723 \mathrm{~m}^{2} /$ day.

The agriculture is the main economic venture of this area. Two distinct cropping seasons, namely Kharif and Rabi, can be seen in the study area. Crops grown during the Kharif season include cotton, paddy, moong, guar, jowar, maize and groundnut. Similarly, wheat, gram, mustard, barley, sugarcane are grown during the Rabi season. 


\section{Methodology}

A total of 300 groundwater samples were collected from privately owned hand pumps in canal catchment areas of Indira Gandhi (site 1), Bhakra (site 2) and Gang (site 3) during the pre-monsoon season. The geographical position and other details of sampling locations are presented in Fig. 1. Samples were collected after $30 \mathrm{~min}$ of pumping and transferred into pre-cleaned polyethylene bottles of 1-L capacity and immediately measured for electrical conductivity (EC), $\mathrm{pH}$ and total dissolved solids (TDS) by digital meters. The collected samples were stored in iceboxes after filtration and brought to the laboratory within $24 \mathrm{~h}$ and then stored in a freezer at $4{ }^{\circ} \mathrm{C}$ for further chemical analysis. Water samples were analyzed for various chemical constituents using standard methods prescribed by the American Public Health Association (APHA 1989, 1995). Calcium $\left(\mathrm{Ca}^{+2}\right)$, magnesium $\left(\mathrm{Mg}^{+2}\right)$ were determined by titrimetric method and sodium $\left(\mathrm{Na}^{+}\right)$, potassium $\left(\mathrm{K}^{+}\right)$by flame photometrically. Chloride $\left(\mathrm{Cl}^{-}\right)$and bicarbonate $\left(\mathrm{HCO}_{3}^{-}\right)$were analyzed titrimetrically, whereas fluoride $\left(\mathrm{F}^{-}\right)$, nitrate $\left(\mathrm{NO}_{3}{ }^{-}\right)$and sulfate $\left(\mathrm{SO}_{4}^{-2}\right)$ were determined by spectrophotometric techniques.

Various irrigation water quality parameters such as total hardness (TH), sodium adsorption ratio (SAR), percent sodium (\%Na), residual sodium carbonate (RSC), residual sodium bicarbonate (RSBC), magnesium hazard (MH), $\mathrm{Mg}$ / Ca ratio, Kelley's index (KI), permeability index (PI) and potential salinity (PS) were calculated using physicochemical analyses (Todd 1980; Hem 1985).

Total Hardness is calculated by the formula- $\mathrm{TH}$

$$
=2.497 \mathrm{Ca}^{+2}+4.115 \mathrm{Mg}^{+2}
$$

The correlation of analytical data and classification for suitability were assessed by plotting different graphical representations like Piper (1944), Wilcox (1955), USSL (1954),
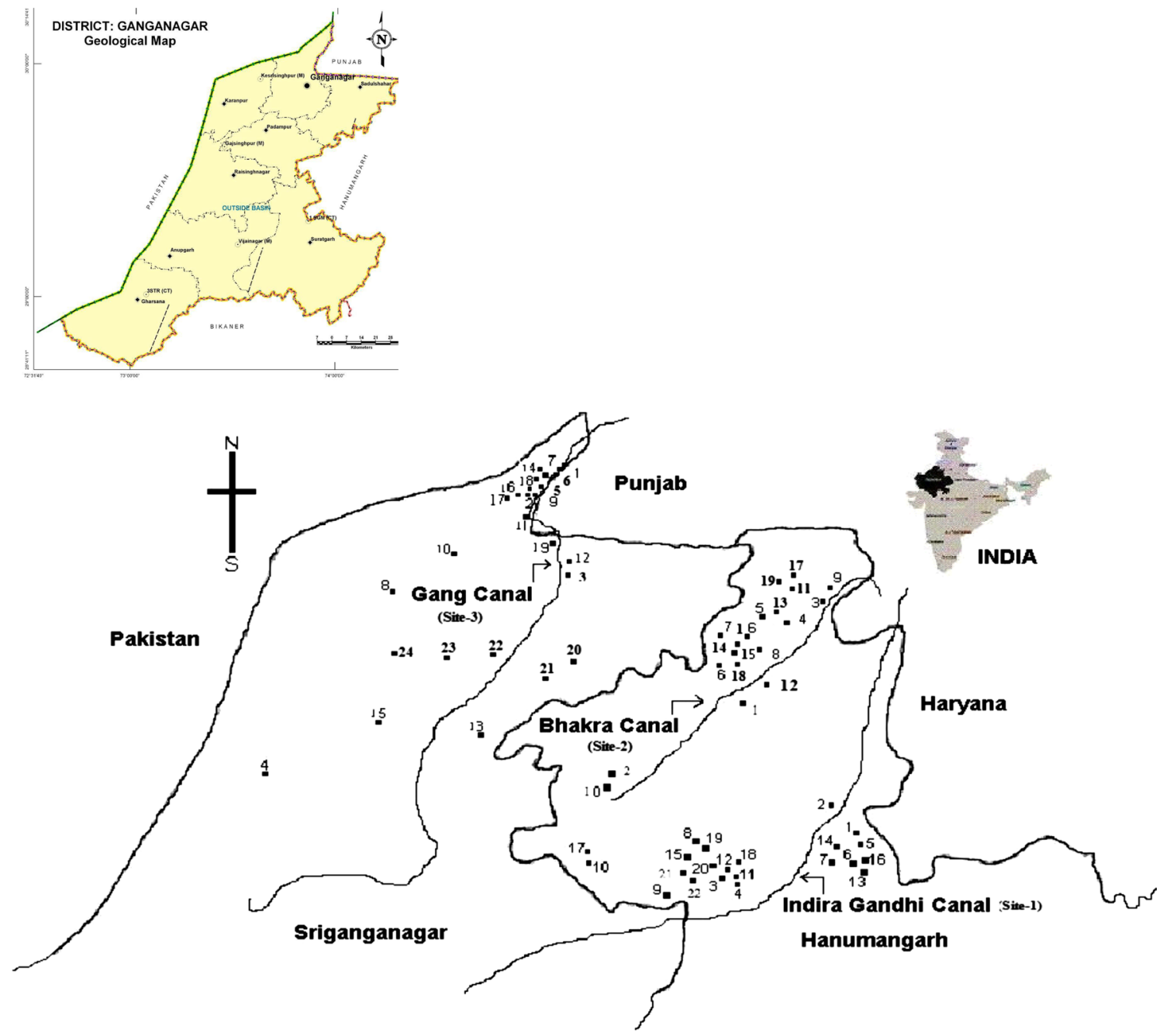

Fig. 1 Location of groundwater sampling in three different sites 
Gibbs (1970), Doneen (1964a) using Arc-GIS-9.3 and rockworks 16 software.

\section{Result and discussion}

Quantitative analyses of different physicochemical parameters are the prime factors to determine the quality of water. Analytical data for the various parameters are given in Tables 1, 2 and 3 for three different sites. To ascertain the suitability of collected groundwater samples for drinking and irrigation purposes was discussed separately on comparison with recommended standard values.

\section{Drinking suitability}

The adequacy of groundwater for domestic use is directly related to different physiochemical parameters and their concentrations. The mean values of different physicochemical parameters for three canal catchment areas are presented in Tables 1, 2 and 3. The $\mathrm{pH}$ of groundwater of all samples ranged between 7.0 and 9.62, which looks slightly higher than the limit described by WHO (6.5-8.5) (WHO 2011). The maximum $\mathrm{pH}$ value $(9.62 \pm 0.27)$ was observed in the Paliwal-12 STB in Indira Gandhi Canal catchment area. All the water samples were designated to be neutral to alkaline in nature. Dissolution of gypsum present in aquifer rock is responsible for higher salinity.

$\mathrm{CaSO}_{4} \cdot 2 \mathrm{H}_{2} \mathrm{O} \leftrightarrow \mathrm{Ca}^{+2}+\mathrm{SO}_{4}^{-2}$

The electrical conductivity (EC) of groundwater was in the range of $310-17,900 \mu \mathrm{S} / \mathrm{cm}$. Most of the samples exceed the permissible level ( $3000 \mu \mathrm{S} / \mathrm{cm}$ BIS 2003) of EC. The sample collected from 56 LNP showed maximum EC $(17,900 \pm 5630)$ which directly indicates the presence of higher levels of cations and anions. The TDS values were remarkably higher and ranged between 219.88 and $6897.29 \mathrm{mg} / \mathrm{l}$ (56 LNP, Gang catchment). The samples collected from Gang Canal catchment had comparatively higher values of TDS which directly indicate the presence of high concentration of soluble salts and make water unsuitable for drinking (Hem 1985). The excess TDS in groundwater enriches the soil salinity and also affects the physical properties and fertility of soil (Table 4).

Davis and De Wiest (1966) classified the water samples under different TDS conditions. In the study, most of water samples belong to hazardous level of TDS for domestic use of groundwater (Table 5).

Total hardness $(\mathrm{TH})$ of groundwater in this area was in the range of 35-4210 $\mathrm{mg} / \mathrm{l}$, and maximum reported sample was from 56 LNP in Gang Canal catchment.
The groundwater study for total hardness (TH) shows that the majority of the samples fall under the hard water category (Table 6).

The hardness of water is due to the presence of alkaline earths such as calcium and magnesium and exists in bicarbonate forms.

The $\mathrm{Mg}^{+2}$ and $\mathrm{Ca}^{+2}$ are important factors of water hardness, and their presence remarkably influences the chemistry of groundwater. The $\mathrm{Mg}^{+2}$ concentration varied from 6.0 to $692.4 \mathrm{mg} / \mathrm{l}$ (maximum in $56 \mathrm{LNP}$, Gang catchment), and $\mathrm{Ca}^{+2}$ ranged from 2.0 to $530 \mathrm{mg} / \mathrm{l}$ (maximum in Jaloki village in Gang catchment). The presence of $\mathrm{Mg}^{+2}$ and $\mathrm{Ca}^{+2}$ in the study area is mainly geogenic in origin as the aquifer of this region is composed of rocks rich in limestone, dolomite and gypsum which contain considerable amount of $\mathrm{Mg}^{+2}$ and $\mathrm{Ca}^{+2}$. Most sampling sites showed remarkable two- to threefold higher concentration of $\mathrm{Mg}^{+2}$ and $\mathrm{Ca}^{+2}$ in groundwater than the safe limit by WHO. Dissolution of gypsum and weathering reactions are responsible for $\mathrm{Ca}^{+2}$ enrichment in groundwater in the region as these minerals found in aquifer rocks.

$\mathrm{CaSO}_{4} \cdot 2 \mathrm{H}_{2} \mathrm{O} \leftrightarrow \mathrm{Ca}^{+2}+\mathrm{SO}_{4}^{-2}$

$\mathrm{CaCO}_{3}+\mathrm{H}_{2} \mathrm{CO}_{3} \leftrightarrow \mathrm{Ca}^{+2}+2 \mathrm{HCO}_{3}^{-}$

Further instantaneous complexing reactions occur.

$\mathrm{Ca}^{+2}+\mathrm{HCO}_{3}^{-} \leftrightarrow \mathrm{CaHCO}_{3}^{+}$

The solubility of Ca carbonate is increased by the formation of the aqueous $\mathrm{Ca}$ bicarbonate complex.

Several epidemiological investigations have demonstrated the relation between risk of cardiovascular disease, growth retardation, reproductive failure and other health problems and hardness of drinking water (Sengupta 2013).

The range of $\mathrm{Na}^{+}$and $\mathrm{K}^{+}$in the area varied from 11.5 to $1009.7 \mathrm{mg} / \mathrm{l}$ and 1.56 to $95.94 \mathrm{mg} / \mathrm{l}$, respectively. Eighty percent of samples collected from Bhakra Canal catchment were exceeding the permissible limit of BIS $200 \mathrm{mg} / \mathrm{l} \mathrm{Na}{ }^{+}$, and maximum was reported in Bolanwali village in Bhakra catchment, whereas groundwater samples in Gang catchment were reported with higher levels of $\mathrm{K}^{+}$with maximum in $56 \mathrm{LNP}$. Although $\mathrm{Na}^{+}$and $\mathrm{K}^{+}$are naturally occurring ions in groundwater, domestic and industrial wastes are other contributors of these (Garg et al. 2009). Cation exchange reaction between $\mathrm{Ca}^{+2}$ and $\mathrm{Na}^{+}$also occurs naturally in the groundwater.

$2 \mathrm{NaX}+\mathrm{Ca}^{+2} \leftrightarrow \mathrm{CaX}_{2}+2 \mathrm{Na}^{+}$

The relationship between elevated sodium intake and hypertension has been the subject of considerable scientific controversy. Excessive salt intake seriously aggravates chronic congestive heart failure, and ill effects due to high 
Table 1 Analytical results (mean values) of groundwater samples in Indira Gandhi Canal catchment area (site 1)

\begin{tabular}{|c|c|c|c|c|c|c|c|c|}
\hline Sample ID & $\begin{array}{l}\text { No. of } \\
\text { samples }\end{array}$ & $\begin{array}{l}\text { Turbidity } \\
\text { (NTU) }\end{array}$ & $\mathrm{pH}$ & $\mathrm{EC}(\mu \mathrm{S} / \mathrm{cm})$ & TDS (mg/l) & TH (mg/l) & $\mathrm{Ca}(\mathrm{mg} / \mathrm{l})$ & $\mathrm{Mg}(\mathrm{mg} / \mathrm{l})$ \\
\hline IGC1 & 5 & 2.54 & $8.05(0.12)$ & $1630(221)$ & $656.28(12.8)$ & 383 (76.4) & $55.6(5.9)$ & $58.56(5.6)$ \\
\hline IGC2 & 6 & 2.65 & $7.79(0.23)$ & $2490(243)$ & $1423.37(304.1)$ & 1153 (43.9) & $158.4(12.8)$ & $181.56(17.8)$ \\
\hline IGC3 & 4 & 0.55 & $7.79(0.07)$ & $2490(345)$ & $1076.91(211.7)$ & 849 (32.9) & $123.6(8.9)$ & $129.6(23.7)$ \\
\hline IGC4 & 11 & 1.40 & $7.66(0.09)$ & $2840(543)$ & $1117.80(226.8)$ & $694(78.8)$ & $98.6(7.9)$ & $107.28(11.2)$ \\
\hline IGC5 & 7 & 3.93 & $8.40(0.12)$ & $2040(256)$ & $1650.57(554.0)$ & $291(43.2)$ & $50.8(4.7)$ & $39.24(4.5)$ \\
\hline IGC6 & 6 & 3.20 & $8.16(0.23)$ & $2110(265)$ & $1073.32(32.9)$ & 297 (22.4) & $48(10.2)$ & $42.36(3.2)$ \\
\hline IGC7 & 5 & 1.86 & $7.74(0.14)$ & $3510(231)$ & $1517.72(508.9)$ & 865 (32.2) & $118.4(16.8)$ & $136.56(23.5)$ \\
\hline IGC8 & 2 & 1.90 & $7.90(0.06)$ & $1370(435)$ & $658.84(34.7)$ & $380(32.8)$ & $68(8.9)$ & $50.4(8.7)$ \\
\hline IGC9 & 3 & 4.40 & $7.70(0.32)$ & $2630(658)$ & $1033.33(65.6)$ & $572(22.1)$ & 85.4 (7.6) & 86.04 (7.8) \\
\hline IGC10 & 3 & 1.00 & $8.86(0.11)$ & $1450(112)$ & $697.32(54.9)$ & $385(44.8)$ & $28.6(4.6)$ & $44.76(7.1)$ \\
\hline IGC11 & 7 & 3.26 & $7.63(0.10)$ & $3250(332)$ & $1359.02(112.9)$ & $1287(112)$ & $198.2(32.1)$ & $189.72(28.7)$ \\
\hline IGC12 & 4 & 2.95 & $7.69(0.04)$ & $1990(521)$ & $884.11(45.7)$ & 854 (56.9) & $168.6(18.2)$ & $103.8(32.4)$ \\
\hline IGC13 & 5 & 2.86 & $7.66(0.02)$ & $3480(332)$ & $1467.04(77.8)$ & $1020(67.9)$ & $158.4(22.1)$ & $149.76(23.3)$ \\
\hline IGC14 & 2 & 1.25 & $7.95(0.07)$ & $1370(554)$ & $567.05(67.6)$ & $405(81.0)$ & $54(8.9)$ & $64.8(4.5)$ \\
\hline IGC15 & 3 & 4.83 & $7.80(0.32)$ & $2340(461)$ & $979.43(34.9)$ & 853 (34.6) & $134.6(21.2)$ & $123.96(36.7)$ \\
\hline IGC16 & 6 & 2.25 & $7.74(0.22)$ & $2290(428)$ & $1627.71(66.5)$ & 1148 (56.6) & $173.4(10.9)$ & $171.36(45.7)$ \\
\hline IGC17 & 5 & 1.96 & $7.60(0.15)$ & $3320(321)$ & $1395.08(69.6)$ & 825 (78.5) & $125.2(8.7)$ & $122.88(34.4)$ \\
\hline IGC18 & 5 & 1.36 & $7.55(0.02)$ & $4940(673)$ & 2051.23 (341.6) & $1345(34.8)$ & $196(54.1)$ & $205.2(32.6)$ \\
\hline IGC19 & 2 & 0.75 & $7.75(0.09)$ & $2430(323)$ & $1044.99(76.6)$ & $550(44.5)$ & $95(4.8)$ & 75 (12.7) \\
\hline IGC20 & 6 & 1.70 & $8.25(0.07)$ & $610(554)$ & $334.36(41.0)$ & $178(66.7)$ & $18(2.3)$ & $31.8(6.9)$ \\
\hline IGC21 & 2 & 1.90 & $7.30(0.16)$ & $4000(448)$ & $1725.78(104.8)$ & $1253(77.8)$ & $222(76.9)$ & $167.4(55.3)$ \\
\hline IGC22 & 2 & 1.70 & $7.40(0.32)$ & $3800(221)$ & $1724.27(102.7)$ & 1300 (110.9) & $218(78.8)$ & $181.2(32.1)$ \\
\hline Sample ID & $\begin{array}{l}\text { No. of } \\
\text { samples }\end{array}$ & $\mathrm{Na}(\mathrm{mg} / \mathrm{l})$ & $\mathrm{K}(\mathrm{mg} / \mathrm{l})$ & $\mathrm{F}(\mathrm{mg} / \mathrm{l})$ & $\mathrm{Cl}(\mathrm{mg} / \mathrm{l})$ & $\mathrm{HCO}_{3}(\mathrm{mg} / \mathrm{l})$ & $\mathrm{NO}_{3}(\mathrm{mg} / \mathrm{l})$ & $\mathrm{SO}_{4}(\mathrm{mg} / \mathrm{l})$ \\
\hline IGC1 & 5 & $96.6(8.9)$ & $10.14(3.4)$ & $4.40(2.3)$ & $162.59(43.6)$ & $378.2(17.8)$ & $25.08(6.7)$ & $56.16(10.8)$ \\
\hline IGC2 & 6 & $207.92(12.3)$ & $18.33(6.7)$ & $4.58(2.1)$ & $511.91(43.2)$ & $477.63(23.8)$ & $25.59(7.6)$ & $78.72(6.8)$ \\
\hline IGC3 & 4 & $119.6(14.5)$ & $15.99(5.4)$ & $5.0(0.6)$ & $348.965(22.5)$ & 411.75 (16.9) & $23.26(4.9)$ & $108.96(21.5)$ \\
\hline IGC4 & 11 & $244.49(54.2)$ & $16.77(10.6)$ & $3.81(0.8)$ & $353.225(33.3)$ & 427 (31.8) & $25.56(5.9)$ & 70.08 (7.8) \\
\hline IGC5 & 7 & $260.59(23.7)$ & $6.63(9.8)$ & $3.50(0.6)$ & $223.65(43.1)$ & $610(76.7)$ & $33.03(4.5)$ & $51.36(6.5)$ \\
\hline IGC6 & 6 & $284.97(45.5)$ & $6.63(6.7)$ & $3.08(1.2)$ & $354.29(22.8)$ & $447.13(65.5)$ & $35.32(6.7)$ & $39.36(5.6)$ \\
\hline IGC7 & 5 & $273.01(54.8)$ & $19.11(6.8)$ & $3.20(2.1)$ & $646.81(34.8)$ & $463.6(8.9)$ & $19.24(6.6)$ & $71.52(7.8)$ \\
\hline IGC8 & 2 & 91.54 (43.7) & $8.58(2.7)$ & $2.50(0.9)$ & $136.675(21.8)$ & $427(43.3)$ & $21.80(3.5)$ & $68.16(10.4)$ \\
\hline IGC9 & 3 & $177.79(32.9)$ & $16.38(3.5)$ & $3.17(0.5)$ & $308.85(56.6)$ & $589.87(56.6)$ & $19.67(6.5)$ & $43.2(8.3)$ \\
\hline IGC10 & 3 & $120.06(34.2)$ & $17.94(4.5)$ & $2.50(0.3)$ & $169.335(23.2)$ & 406.87 (12.9) & $17.50(7.8)$ & $15.36(7.5)$ \\
\hline IGC11 & 7 & $158.24(22.1)$ & $16.38(7.6)$ & $2.79(0.4)$ & 489.545 (33.6) & $383.69(15.7)$ & $17.48(4.5)$ & $95.04(13.5)$ \\
\hline IGC12 & 4 & $48.53(23.4)$ & $10.53(5.2)$ & $2.75(0.5)$ & $304.59(43.2)$ & $320.25(21.7)$ & $10.63(7.5)$ & $76.32(10.2)$ \\
\hline IGC13 & 5 & $243.8(35.5)$ & $15.21(4.4)$ & $2.80(0.3)$ & $614.15(54.2)$ & $378.2(54.8)$ & $26.86(10.4)$ & $68.16(8.7)$ \\
\hline IGC14 & 2 & $74.29(31.8)$ & $8.97(5.7)$ & $5.25(0.4)$ & $86.975(22.8)$ & $366(65.6)$ & $23.25(6.8)$ & $71.04(5.2)$ \\
\hline IGC15 & 3 & $113.62(32.6)$ & $10.92(7.2)$ & $3.50(0.6)$ & 307.785 (43.8) & 386.13 (34.6) & $13.79(8.5)$ & $80.16(14.5)$ \\
\hline IGC16 & 6 & $317.63(22.8)$ & $16.77(6.9)$ & $3.17(0.4)$ & $561.61(25.5)$ & 427 (76.6) & $25.23(6.6)$ & $133.92(32.1)$ \\
\hline IGC17 & 5 & $252.31(45.6)$ & $10.92(8.2)$ & $2.60(0.6)$ & $541.73(43.2)$ & $414.8(43.3)$ & $30.23(7.8)$ & $102.72(11.6)$ \\
\hline IGC18 & 5 & $386.4(43.8)$ & $17.94(5.9)$ & $3.90(0.5)$ & $803.72(56.6)$ & $512.4(21.3)$ & $20.36(5.6)$ & $163.2(12.5)$ \\
\hline IGC19 & 2 & $178.71(45.7)$ & $11.7(5.7)$ & $2.75(0.2)$ & $397.6(22.7)$ & $427(56.8)$ & $23.28(4.9)$ & 49.44 (5.7) \\
\hline IGC20 & 6 & $47.15(7.8)$ & $9.36(3.5)$ & $1.92(0.7)$ & $72.065(8.7)$ & $203.13(21.5)$ & $20.30(7.8)$ & $11.04(8.5)$ \\
\hline IGC21 & 2 & $192.74(19.5)$ & $12.48(2.2)$ & $4.50(0.5)$ & 704.675 (18.9) & $488(48.8)$ & $24.40(9.5)$ & $156.48(4.6)$ \\
\hline IGC22 & 2 & $155.25(43.3)$ & $12.87(3.2)$ & $2.50(0.5)$ & $392.275(34.8)$ & $488(34.9)$ & $25.20(4.8)$ & $140.16(23.8)$ \\
\hline
\end{tabular}


Table 2 Analytical results (mean values) of groundwater samples in Bhakra Canal catchment area (site 2)

\begin{tabular}{|c|c|c|c|c|c|c|c|c|}
\hline Sample ID & $\begin{array}{l}\text { No. of } \\
\text { samples }\end{array}$ & $\begin{array}{l}\text { Turbidity } \\
\text { (NTU) }\end{array}$ & $\mathrm{pH}$ & $\mathrm{EC}(\mu \mathrm{S} / \mathrm{cm})$ & TDS (mg/l) & $\mathrm{TH}(\mathrm{mg} / \mathrm{l})$ & $\mathrm{Ca}(\mathrm{mg} / \mathrm{l})$ & $\mathrm{Mg}(\mathrm{mg} / \mathrm{l})$ \\
\hline $\mathrm{BC} 1$ & 8 & 2.03 & $8.01(1.45)$ & $2220(443)$ & $946.44(34.2)$ & $655(34.8)$ & $61.2(3.5)$ & $120.48(32.8)$ \\
\hline $\mathrm{BC} 2$ & 7 & 5.40 & $7.63(2.23)$ & $3870(456)$ & $1661.91(156.2)$ & $684.29(56.9)$ & $112.6(4.8)$ & $96.72(5.4)$ \\
\hline $\mathrm{BC} 3$ & 2 & 2.0 & $7.84(0.87)$ & $8050(832)$ & $2770.66(234.0)$ & $1525(234)$ & $238(23.7)$ & $223.2(32.7)$ \\
\hline $\mathrm{BC} 4$ & 8 & 6.83 & $7.92(0.56)$ & $5400(435)$ & $2185.11(78.8)$ & $1051.25(78.9)$ & $170.2(34.4)$ & $150.12(6.7)$ \\
\hline BC5 & 8 & 4.03 & $7.86(0.76)$ & $4500(675)$ & $1988.05(242)$ & $844.38(23.8)$ & $139.6(12.7)$ & $118.92(15.6)$ \\
\hline BC6 & 3 & 0.97 & $8.55(1.20)$ & $3070(342)$ & 1494.57 (554) & 450 (45.9) & $63.4(6.5)$ & $69.96(7.8)$ \\
\hline BC7 & 3 & 5.60 & $8.37(0.05)$ & $1940(128)$ & $957.01(45.9)$ & $275(34.5)$ & $47.4(2.8)$ & $37.56(8.4)$ \\
\hline BC8 & 5 & 2.52 & $7.39(0.32)$ & $4190(654)$ & $1718.95(98.7)$ & $1122(198)$ & $175.6(34.7)$ & $163.92(10.6)$ \\
\hline BC9 & 6 & 1.58 & $7.42(0.45)$ & $3690(823)$ & $1650.37(38.8)$ & 946.67 (82.9) & $168.4(33.5)$ & $126.24(12.3)$ \\
\hline $\mathrm{BC} 10$ & 6 & 1.22 & $8.17(0.87)$ & $6280(467)$ & $2560.47(165)$ & $1154(231)$ & $145.6(22.1)$ & $189.6(10.3)$ \\
\hline BC11 & 2 & 1.0 & $8.51(0.34)$ & $4550(342)$ & $2061.04(210)$ & 1103 (59.9) & $182(38.8)$ & $155.4(7.7)$ \\
\hline $\mathrm{BC} 12$ & 5 & 1.20 & $7.88(0.78)$ & $7620(321)$ & 2922.35 (154) & 2064 (178) & $197.2(56.6)$ & $377.04(14.5)$ \\
\hline BC13 & 2 & 4.60 & $7.46(0.34)$ & $6350(228)$ & $2695.88(235)$ & $1313(58.9)$ & $100(34.8)$ & 255 (12.6) \\
\hline BC14 & 4 & 1.80 & $7.89(0.65)$ & $4380(765)$ & $1898.40(345)$ & $936.25(45.6)$ & $128.6(12.4)$ & $147.6(16.8)$ \\
\hline BC15 & 7 & 3.87 & $7.98(0.87)$ & $5490(389)$ & $2229.89(231)$ & $1246(342)$ & $184.2(34.2)$ & $188.52(23.7)$ \\
\hline BC16 & 2 & 8.35 & $7.48(0.22)$ & $6650(548)$ & $2596.20(174)$ & $1133(278)$ & $182(56.2)$ & $162.6(21.4)$ \\
\hline BC17 & 15 & 3.46 & 7.80 (1.78). & $3920(387)$ & $1783.14(78.9)$ & $804.67(56.3)$ & $138.2(16.7)$ & $110.16(24.8)$ \\
\hline BC18 & 4 & 9.68 & $8.0(0.45)$ & $2120(389)$ & $966.89(56.7)$ & $433.75(23.8)$ & $74(7.8)$ & $59.76(5.7)$ \\
\hline BC19 & 3 & 1.57 & $8.06(0.34)$ & $3290(438)$ & $1408.81(145)$ & 595 (38.9) & $99.4(5.9)$ & $83.16(6.7)$ \\
\hline Sample ID & $\begin{array}{l}\text { No. of } \\
\text { samples }\end{array}$ & $\mathrm{Na}(\mathrm{mg} / \mathrm{l})$ & $\mathrm{K}(\mathrm{mg} / \mathrm{l})$ & $\mathrm{F}(\mathrm{mg} / \mathrm{l})$ & $\mathrm{Cl}(\mathrm{mg} / \mathrm{l})$ & $\mathrm{HCO}_{3}(\mathrm{mg} / \mathrm{l})$ & $\mathrm{NO}_{3}(\mathrm{mg} / \mathrm{l})$ & $\mathrm{SO}_{4}(\mathrm{mg} / \mathrm{l})$ \\
\hline $\mathrm{BC} 1$ & 8 & $185.38(23.4)$ & $12.87(5.6)$ & $1.69(0.6)$ & $206.965(45.8)$ & $411.75(23.8)$ & $7.06(0.6)$ & $115.2(23.2)$ \\
\hline $\mathrm{BC} 2$ & 7 & $423.66(22.8)$ & $11.31(3.4)$ & $4.36(0.7)$ & $621.605(36.8)$ & $496.54(24.5)$ & $10.15(0.5)$ & $138.72(13.7)$ \\
\hline $\mathrm{BC} 3$ & 2 & $817.19(45.5)$ & $19.89(6.5)$ & $5.75(0.5)$ & 1031.275 (76.6) & $518.5(33.8)$ & $5.72(0.7)$ & $174.24(24.5)$ \\
\hline $\mathrm{BC} 4$ & 8 & $558.9(34.4)$ & $11.7(4.5)$ & $2.38(1.0)$ & $878.625(56.6)$ & 488 (56.6) & $12.31(1.7)$ & $157.92(32.7)$ \\
\hline BC5 & 8 & $515.43(43.3)$ & $11.7(4.8)$ & $4.31(0.9)$ & $796.62(78.8)$ & $465.43(37.7)$ & $9.83(1.5)$ & $147.84(14.5)$ \\
\hline BC6 & 3 & $465.98(54.4)$ & $8.97(2.3)$ & $3.67(0.5)$ & 488.835 (45.9) & $528.87(26.6)$ & $11.13(1.9)$ & $146.4(6.9)$ \\
\hline $\mathrm{BC7}$ & 3 & $275.77(23.3)$ & $9.75(3.7)$ & $1.50(0.6)$ & $185.665(23.8)$ & $528.87(45.5)$ & $11.44(0.9)$ & $64.8(5.9)$ \\
\hline BC8 & 5 & $326.14(62.7)$ & $18.72(2.5)$ & $1.90(0.4)$ & $587.88(56.6)$ & $585.6(42.2)$ & $10.64(0.7)$ & $141.6(10.8)$ \\
\hline BC9 & 6 & $306.13(75.5)$ & $9.75(3.2)$ & $1.0(0.5)$ & $565.16(45.5)$ & $599.63(12.8)$ & $14.84(0.7)$ & $158.88(7.6)$ \\
\hline BC10 & 6 & $727.26(45.5)$ & $18.72(3.0)$ & $3.0(0.9)$ & $1021.69(33.6)$ & $477.63(43.9)$ & $19.43(0.8)$ & $157.92(10.5)$ \\
\hline BC11 & 2 & $540.96(76.6)$ & $15.6(2.4)$ & $2.75(0.5)$ & $793.425(23.8)$ & $335.5(23.3)$ & $3.53(0.3)$ & $141.12(9.8)$ \\
\hline BC12 & 5 & $661.71(34.8)$ & $24.18(4.6)$ & $3.20(0.8)$ & $1249.6(45.5)$ & $463.6(45.8)$ & $11.09(2.8)$ & $166.08(32.6)$ \\
\hline $\mathrm{BC} 13$ & 2 & $694.83(47.8)$ & $17.55(3.8)$ & $2.75(0.6)$ & $1221.2(43.1)$ & 427 (37.8) & $14.12(0.8)$ & $177.6(12.0)$ \\
\hline BC14 & 4 & $516.81(23.3)$ & $14.43(5.6)$ & $3.38(0.5)$ & $684.44(65.7)$ & $457.5(45.5)$ & $4.98(1.2)$ & $156.48(21.7)$ \\
\hline BC15 & 7 & $507.15(34.2)$ & $18.33(4.2)$ & $3.0(0.7)$ & $928.68(33.2)$ & 427 (67.7) & $22.19(6.8)$ & $148.32(21.5)$ \\
\hline BC16 & 2 & $720.13(62.8)$ & $16.77(2.3)$ & $2.75(0.5)$ & $1128.9(123.8)$ & $396.5(34.8)$ & $4.88(0.6)$ & $181.92(11.5)$ \\
\hline $\mathrm{BC} 17$ & 15 & $446.2(66.7)$ & $11.31(7.2)$ & $3.13(0.6)$ & $663.495(32.8)$ & $483.73(34.8)$ & $13.85(4.7)$ & $153.6(12.3)$ \\
\hline BC18 & 4 & $243.34(34.5)$ & $9.36(2.4)$ & $3.63(0.9)$ & $295.715(57.7)$ & $381.25(23.9)$ & $12.00(3.8)$ & $82.08(4.9)$ \\
\hline BC19 & 3 & $368.46(54.2)$ & $7.8(3.1)$ & $4.50(0.7)$ & $383.4(42.1)$ & $589.87(32.2)$ & $11.51(7.8)$ & $139.2(7.2)$ \\
\hline
\end{tabular}

levels of sodium in drinking water have been documented. Infants with severe gastrointestinal infections can suffer from fluid loss, leading to dehydration and raised sodium levels in the plasma (hypernatraemia); permanent neurological damage is common under such conditions.

Fluoride toxicity due to dissolution of fluoride containing rocks was a major concern in the area and few samples were reported for nitrate toxicity. One of the essential elements for maintaining normal development of healthy teeth and bones is fluoride. Lower concentrations of fluoride usually below $0.6 \mathrm{mg} / \mathrm{l}$ may contribute to dental caries. However, continuing consumption of higher concentrations above $1.5 \mathrm{ppm}$ may cause dental fluorosis and in extreme cases even skeletal fluorosis. Concentrations of fluoride in samples 
Table 3 Analytical results (mean values) of groundwater samples in Gang Canal catchment area (site 3)

\begin{tabular}{|c|c|c|c|c|c|c|c|c|}
\hline Sample ID & $\begin{array}{l}\text { No. of } \\
\text { samples }\end{array}$ & Turbidity (NTU) & $\mathrm{pH}$ & $\mathrm{EC}(\mu \mathrm{S} / \mathrm{cm})$ & TDS (mg/l) & TH (mg/l) & $\mathrm{Ca}(\mathrm{mg} / \mathrm{l})$ & $\mathrm{Mg}(\mathrm{mg} / \mathrm{l})$ \\
\hline GC1 & 5 & 2.94 & $7.57(0.6)$ & $2980(654)$ & $1297.39(321)$ & 815 (32.7) & $186.4(34.6)$ & $141.12(26.6)$ \\
\hline GC2 & 6 & 3.27 & $7.40(1.2)$ & $2480(324)$ & $1092.11(276)$ & $748(22.0)$ & $138(67.0)$ & $96.84(8.9)$ \\
\hline GC3 & 8 & 2.29 & $7.20(0.5)$ & $5450(482)$ & 2120.09 (437) & $1451(410)$ & $283(34.3)$ & $178.56(23.7)$ \\
\hline GC4 & 6 & 13.72 & $7.46(0.4)$ & $2880(536)$ & 1193.71 (127) & $743(45.0)$ & $127(20.2)$ & $102.24(12.6)$ \\
\hline GC5 & 6 & 1.50 & $7.27(0.8)$ & $5680(348)$ & $2281.56(427)$ & $1656.67(321)$ & $276.6(12.8)$ & $231.6(23.5)$ \\
\hline GC6 & 3 & 1.93 & $7.32(0.8)$ & $9270(265)$ & 3365.22 (437) & 2123 (128) & $317.4(67.7)$ & $319.2(16.8)$ \\
\hline GC7 & 3 & 2.13 & $7.46(0.9)$ & 7270 (276) & $2885.36(387)$ & 1867 (328) & $316.6(33.9)$ & $258(18.8)$ \\
\hline GC8 & 1 & 3.0 & 7.78 & 4800 & 1937.19 & 1660 & 336 & 196.8 \\
\hline GC9 & 3 & 5.57 & $7.33(0.6)$ & 3720 (328) & $1589.46(606)$ & $1180(456)$ & $220(45.5)$ & $151.2(23.1)$ \\
\hline GC10 & 2 & 11.95 & $7.63(0.5)$ & $2080(623)$ & $971.48(57.9)$ & $685(43.3)$ & 109 (10.9) & $99(7.8)$ \\
\hline GC11 & 2 & 14.05 & $7.53(0.8)$ & $5050(432)$ & 2326.85 (307) & $1625(421)$ & $292(56.9)$ & $214.8(34.4)$ \\
\hline GC12 & 2 & 3.2 & $7.48(0.6)$ & 4650 (610) & $2063.44(653)$ & $1460(180)$ & 277 (32.9) & $184.2(22.1)$ \\
\hline GC13 & 2 & 2.05 & $7.67(0.8)$ & $11,750(724)$ & $4305.96(287)$ & 2915 (203) & $529(27.8)$ & $382.2(17.8)$ \\
\hline GC14 & 4 & 15.78 & $7.28(0.7)$ & 2000 (109) & $934.98(453)$ & 740 (58.9) & $141.6(67.7)$ & $92.76(8.7)$ \\
\hline GC15 & 4 & 1.40 & $7.38(0.4)$ & 4160 (287) & 1694.54 (287) & $1097.5(74.8)$ & $202.6(56.6)$ & $141.96(6.7)$ \\
\hline GC16 & 11 & 15.24 & $7.53(0.6)$ & 2260 (876) & $992.13(87.5)$ & $663(43.8)$ & $119.4(20.5)$ & $87.36(8.9)$ \\
\hline GC17 & 3 & 11.13 & $7.75(0.4)$ & $1590(328)$ & $749.49(50.6)$ & $530(45.9)$ & $88.6(10.9)$ & 74.04 (5.6) \\
\hline GC18 & 4 & 4.18 & $7.67(0.7)$ & $5150(554)$ & 2199.48 (586) & 1504 (254) & $294.2(43.3)$ & $199.8(23.4)$ \\
\hline GC19 & 12 & 3.48 & $7.44(0.8)$ & $5770(336)$ & 2297.18 (387) & $1688.33(834)$ & $322.6(17.7)$ & $211.56(10.9)$ \\
\hline GC20 & 2 & 0.35 & $7.58(0.9)$ & 2120 (374) & $1003.06(178)$ & $670(49.9)$ & $124(39.9)$ & $86.4(10.2)$ \\
\hline GC21 & 1 & 14.20 & 7.36 & 4500 & 1873.64 & 1440 & 290 & 171.6 \\
\hline GC22 & 4 & 6.04 & $7.60(0.5)$ & $2760(331)$ & $1220.05(265)$ & $757.50(37.7)$ & $133.6(34.4)$ & $101.76(33.2)$ \\
\hline GC23 & 2 & 0.45 & $7.61(0.7)$ & 9700 (213) & $3827.51(523)$ & 2295 (278) & $336(56.6)$ & $349.2(23.2)$ \\
\hline $\mathrm{GC} 24$ & 4 & 1.85 & $7.74(0.6)$ & 8300 (276) & $3375.76(543)$ & $2150(478)$ & $356(17.9)$ & $302.4(21.9)$ \\
\hline Sample ID & $\begin{array}{l}\text { No. of } \\
\text { samples }\end{array}$ & $\mathrm{Na}(\mathrm{mg} / \mathrm{l})$ & $\mathrm{K}(\mathrm{mg} / \mathrm{l})$ & $\mathrm{F}(\mathrm{mg} / \mathrm{l})$ & $\mathrm{Cl}(\mathrm{mg} / \mathrm{l})$ & $\mathrm{HCO}_{3(\mathrm{mg} / \mathrm{l})}$ & $\mathrm{NO}_{3}(\mathrm{mg} / \mathrm{l})$ & $\mathrm{SO}_{4}(\mathrm{mg} / \mathrm{l})$ \\
\hline GC1 & 5 & $123.74(34.4)$ & $15.21(7.8)$ & $3.50(0.7)$ & $540.31(23.7)$ & $464.82(28.8)$ & $7.38(0.6)$ & 49.44 (1.6) \\
\hline $\mathrm{GC} 2$ & 6 & $193.89(12.6)$ & $13.65(8.0)$ & $2.58(0.5)$ & $436.65(55.0)$ & $306.83(56.6)$ & $11.57(0.5)$ & $47.04(2.3)$ \\
\hline GC3 & 8 & $401.81(23.2)$ & $19.89(8.8)$ & $2.88(0.5)$ & $965.245(28.8)$ & $416.63(12.8)$ & $8.32(0.7)$ & $55.68(2.4)$ \\
\hline GC4 & 6 & $227.01(11.5)$ & $11.7(6.9)$ & $1.50(0.6)$ & $530.015(15.5)$ & $331.23(28.8)$ & $6.62(0.8)$ & $25.92(2.5)$ \\
\hline GC5 & 6 & 272.78 (21.6) & $27.69(6.0)$ & $2.83(0.4)$ & $1204.16(87.9)$ & $341.6(54.9)$ & $5.92(0.5)$ & $90.24(6.5)$ \\
\hline GC6 & 3 & $529(23.3)$ & $21.45(7.9)$ & $2.83(0.4)$ & $1863.75(123)$ & $370.27(39.5)$ & $11.38(0.6)$ & 97.44 (7.8) \\
\hline GC7 & 3 & $463.45(45.5)$ & $21.84(9.8)$ & $2.33(0.7)$ & 1477.865 (109) & 480.07 (81.0) & $9.55(0.5)$ & $95.52(8.0)$ \\
\hline GC8 & 1 & 252.77 & 15.21 & 2.5 & 759.7 & 524.6 & 21.85 & 90.24 \\
\hline GC9 & 3 & $237.13(22.8)$ & $8.58(0.9)$ & $2.5(0.3)$ & $656.75(28.8)$ & $434.93(20.8)$ & $13.58(0.7)$ & $83.04(6.9)$ \\
\hline GC10 & 2 & $130.87(23.3)$ & $8.97(0.8)$ & $2.0(0.7)$ & 392.275 (78.9) & 347.7 (12.9) & $10.15(0.8)$ & $46.56(6.0)$ \\
\hline GC11 & 2 & $332.58(13.5)$ & $11.7(1.2)$ & $2.0(0.4)$ & 1098.725 (56.9) & $536.8(28.8)$ & $11.82(0.7)$ & $95.04(5.7)$ \\
\hline GC12 & 2 & 340.17 (12.8) & $9.75(0.6)$ & $2.25(0.4)$ & 898.15 (39.9) & $463.6(43.3)$ & $29.52(0.4)$ & $91.2(7.9)$ \\
\hline GC13 & 2 & $579.14(22.0)$ & $25.35(2.1)$ & $2.50(0.6)$ & $2488.55(287)$ & 359.9 (28.9) & $7.97(0.6)$ & 110.88 (8.9) \\
\hline GC14 & 4 & $91.08(45.9)$ & $17.16(3.8)$ & $2.63(0.4)$ & $356.065(45.5)$ & $332.45(45.9)$ & $25.74(0.4)$ & $43.68(5.9)$ \\
\hline GC15 & 4 & $339.71(23.5)$ & $14.82(3.6)$ & $1.63(0.2)$ & 739.465 (49.9) & $414.8(29.3)$ & $5.59(0.8)$ & $41.76(6.8)$ \\
\hline GC16 & 11 & $161.92(48.8)$ & $8.97(0.8)$ & $1.77(0.5)$ & $436.65(12.6)$ & $278.16(10.7)$ & $3.93(0.9)$ & $34.56(5.9)$ \\
\hline GC17 & 3 & $95.22(10.1)$ & $5.46(0.5)$ & $1.0(0.6)$ & 294.65 (17.7) & $325.13(43.9)$ & $6.58(0.7)$ & $21.12(5.5)$ \\
\hline GC18 & 4 & $338.33(23.8)$ & $10.92(1.9)$ & $2.38(0.4)$ & $857.325(23.7)$ & $390.4(28.8)$ & $4.32(0.5)$ & $97.44(6.0)$ \\
\hline GC19 & 12 & $338.33(22.4)$ & $13.26(1.7)$ & $2.58(0.6)$ & $1103.695(12.8)$ & $410.53(33.2)$ & $5.10(0.8)$ & $95.52(9.2)$ \\
\hline GC20 & 2 & 133.17 (11.5) & $4.29(0.6)$ & $1.50(0.7)$ & $422.45(17.5)$ & $329.4(43.2)$ & $9.03(0.6)$ & $58.08(4.8)$ \\
\hline GC21 & 1 & 252.77 (11.8) & 11.31 & 3.0 & $766.8(23.2)$ & 536.8 & 20.65 & 90.24 \\
\hline GC22 & 4 & 207.92 (10.6) & $5.07(0.5)$ & $3.13(0.4)$ & $507.65(12.8)$ & $359.9(21.2)$ & $5.19(0.5)$ & $78.24(4.0)$ \\
\hline
\end{tabular}


Table 3 (continued)

\begin{tabular}{llllllrrr}
\hline Sample ID & $\begin{array}{l}\text { No. of } \\
\text { samples }\end{array}$ & $\mathrm{Na}(\mathrm{mg} / \mathrm{l})$ & $\mathrm{K}(\mathrm{mg} / \mathrm{l})$ & $\mathrm{F}(\mathrm{mg} / \mathrm{l})$ & $\mathrm{Cl}(\mathrm{mg} / \mathrm{l})$ & $\mathrm{HCO}_{3}(\mathrm{mg} / \mathrm{l})$ & $\mathrm{NO}_{3}(\mathrm{mg} / \mathrm{l})$ & $\mathrm{SO} 4(\mathrm{mg} / \mathrm{l})$ \\
\hline $\mathrm{GC} 23$ & 2 & $591.79(23.8)$ & $21.45(4.5)$ & $3.25(0.5)$ & $2232.95(432)$ & $353.8(34.8)$ & $6.96(0.8)$ & $109.92(8.9)$ \\
$\mathrm{GC} 24$ & 4 & $427.8(12.8)$ & $30.81(5.8)$ & $1.88(0.4)$ & $1532.18(189)$ & $334.28(23.3)$ & $65.14(245.9)$ & $68.16(6.8)$ \\
\hline
\end{tabular}

Table 4 Groundwater samples of the study area exceeding the recommended permissible limits for drinking purposes

\begin{tabular}{|c|c|c|c|c|c|c|c|c|c|}
\hline \multirow[t]{3}{*}{ Parameter } & \multirow[t]{3}{*}{ BIS (2003) } & \multicolumn{2}{|l|}{ WHO (2004) } & \multicolumn{6}{|c|}{ Range and percent samples exceeding the permissible limit as per BIS } \\
\hline & & \multirow[t]{2}{*}{ Most desirable } & \multirow{2}{*}{$\begin{array}{l}\text { Maximum } \\
\text { allowable }\end{array}$} & \multicolumn{2}{|l|}{ Site 1} & \multicolumn{2}{|l|}{ Site 2} & \multicolumn{2}{|l|}{ Site 3} \\
\hline & & & & Range & $\begin{array}{l}\text { Percent } \\
\text { samples }\end{array}$ & Range & $\begin{array}{l}\text { Percent } \\
\text { samples }\end{array}$ & Range & $\begin{array}{l}\text { Percent } \\
\text { samples }\end{array}$ \\
\hline $\mathrm{pH}$ & $6.5-8.5$ & 6.5 & 8.5 & $7.20-9.62$ & 14 & $7.13-8.74$ & 19 & $7.0-8.01$ & Nil \\
\hline $\mathrm{EC}(\mu \mathrm{S} / \mathrm{cm})$ & 3000 & 1400 & - & $310-9100$ & 41 & $460-13,900$ & 70 & $460-17,900$ & 69 \\
\hline TDS (mg/l) & 2000 & 500 & 1500 & $219.88-2586.97$ & 14 & $307.99-4700.20$ & 39 & $224.2-6897.29$ & 44 \\
\hline TH (mg/l) & 600 & 100 & 500 & $35-1965$ & 57 & $135-2840$ & 69 & $133-4210$ & 73 \\
\hline $\mathrm{Ca}^{+2}(\mathrm{mg} / \mathrm{l})$ & 200 & 75 & 200 & $2.0-332$ & 20 & $4.0-420$ & 19 & $22-530$ & 58 \\
\hline $\mathrm{Mg}^{+2}(\mathrm{mg} / \mathrm{l})$ & 100 & 50 & 150 & $6.0-366.0$ & 49 & $13.2-546$ & 56 & $20.4-692.4$ & 72 \\
\hline $\mathrm{Na}^{+}(\mathrm{mg} / \mathrm{l})$ & 200 & - & 200 & $13.11-804.08$ & 23 & $24.38-1009.7$ & 80 & $11.5-852.38$ & 58 \\
\hline $\mathrm{K}^{+}(\mathrm{mg} / \mathrm{l})$ & 10 & - & 12 & $1.56-26.91$ & 59 & $5.07-31.59$ & 59 & $1.56-95.94$ & 62 \\
\hline $\mathrm{F}^{-}(\mathrm{mg} / \mathrm{l})$ & 1.5 & - & 1.5 & $0.5-8.0$ & 42 & $0.5-8.5$ & 78 & $0.5-5.0$ & 82 \\
\hline $\left.\mathrm{Cl}^{-(} \mathrm{mg} / \mathrm{l}\right)$ & 1000 & 200 & 600 & $24.85-1533.6$ & 7 & $24.85-2403.35$ & 23 & $56.8-4487.2$ & 33 \\
\hline $\mathrm{NO}_{3}^{-}(\mathrm{mg} / \mathrm{l})$ & 45 & 45 & - & $3.70-82.11$ & 5 & $0.34-50.76$ & 1 & $0.50-278.68$ & 2 \\
\hline $\mathrm{SO}_{4}^{-2}(\mathrm{mg} / \mathrm{l})$ & 200 & 200 & 400 & $0.48-188.64$ & Nil & $5.76-190.08$ & Nil & $2.88-116.64$ & Nil \\
\hline Turbidity (NTU) & 10 & - & - & $0.3-8.0$ & Nil & $0.1-25.0$ & 6 & $0.7-60.0$ & 23 \\
\hline
\end{tabular}

Table 5 Classification of groundwater samples based on TDS (Davis and De Wiest 1966)

\begin{tabular}{lllll}
\hline TDS (mg/l) & Water type & \multicolumn{3}{l}{ Percent samples } \\
\cline { 3 - 5 } & & Site 1 & Site 2 & Site 3 \\
\hline$<500$ & Desirable for drinking & 21 & 08 & 14 \\
$500-1000$ & Permissible for drinking & 27 & 12 & 13 \\
$<3000$ & Useful for irrigation & 50 & 67 & 62 \\
$>3000$ & Unfit for drinking and irrigation & 02 & 13 & 11 \\
\hline
\end{tabular}

Table 6 Sawyer and McCarthy's (Sawyer and McMcartly 1967) classification of groundwater based on hardness

\begin{tabular}{lllll}
\hline TH as $\mathrm{CaCO}_{3}(\mathrm{mg} / \mathrm{l})$ & Water class & \multicolumn{3}{l}{ Percent samples } \\
\cline { 3 - 5 } & & Site 1 & Site 2 & Site 3 \\
\hline$<75$ & Soft & 03 & 00 & 00 \\
$75-150$ & Moderately hard & 10 & 03 & 02 \\
$150-300$ & Hard & 13 & 08 & 10 \\
$>300$ & Very hard & 74 & 89 & 88 \\
\hline
\end{tabular}

taken from the study area varied from 0.5 to $8.0 \mathrm{ppm}$ (site 1), 0.5 to $8.5 \mathrm{ppm}$ (site 2) and 0.5 to $5.0 \mathrm{ppm}$ (Site 3) with different mean values as shown in Fig. 2 (Chaudhary et al. 2008, 2009). There may therefore be the likelihood of the risk of dental caries, suffered by people who depend on this water source for drinking. A high rate of evapotranspiration, longer residence time of waters in the aquifer zone, intensive and long-term irrigation and heavy use of fertilizers are the supplementary factors to further increase the $\mathrm{F}^{-}$content in the groundwaters (Subba Rao 2003). Very high levels of $\mathrm{F}^{-}$and other physicochemical parameters were observed and discussed in the study conducted in different villages of Haryana (Meenakshi et al. 2004).

The $\mathrm{Cl}^{-}$content was observed in the range of $24.85-4487.2 \mathrm{mg} / \mathrm{l}$, and maximum was reported in $56 \mathrm{LNP}$ village in Gang catchment. Thirty-three percent of groundwater samples in Gang catchment and 23\% in Bhakra catchment were found with exceeding level of $\mathrm{Cl}^{-}$prescribed by BIS $1000 \mathrm{mg} / \mathrm{l}$. Only 8\% of groundwater samples were having higher concentration of $\mathrm{NO}_{3}{ }^{-}$in all the samples, and maximum $278.68 \mathrm{mg} / \mathrm{l}$ was observed only in one water sample from Bhagwansar-chunavad 31GG (Gang catchment). $\mathrm{NO}_{3}{ }^{-}$is considered relatively non-toxic, but at the higher concentration in drinking water may cause 
Fig. 2 Fluoride concentration (mean values) in three different sites

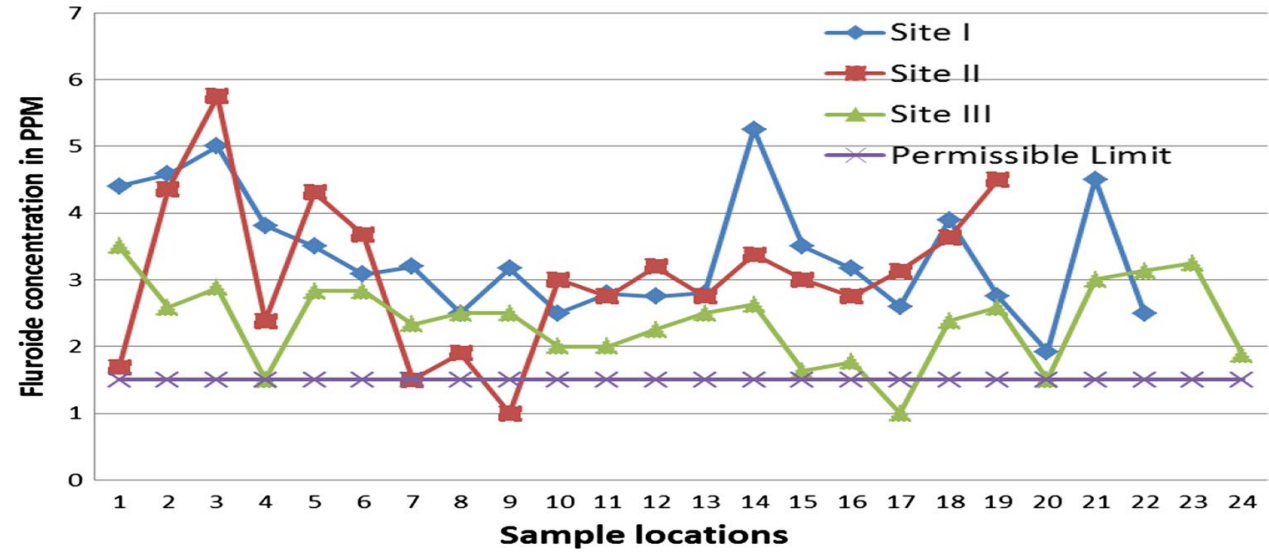

methaemoglobinemia (in infants). Farmers have been using $\mathrm{N}$-fertilizers in their fields, and leaching of $\mathrm{NO}_{3}{ }^{-}$through soil profile is the main reason of nitrate enrichment in the local aquifer as water infiltration rate is comparatively higher in this region (Chaudhary et al. 2010). The $\mathrm{SO}_{4}^{-2}$ content varied from 0.48 to $190.08 \mathrm{mg} / \mathrm{l}$, and all were within the permissible range as decided by WHO $(400 \mathrm{mg} / \mathrm{l})$ and BIS (200 mg/l). $\mathrm{SO}_{4}^{-2}$ in groundwater mainly occurs due to sulfate mineral dissolution and sulfide mineral oxidation (Krouse and Mayer 1999).

The abundance of major ions was found in the following order:

$\mathrm{Na}^{+}>\mathrm{Ca}^{+2}>\mathrm{Mg}^{+2}>\mathrm{K}^{+}=\mathrm{Cl}^{-}>\mathrm{HCO}_{3}^{-}>\mathrm{SO}_{4}^{-2}>\mathrm{NO}_{3}^{-}>\mathrm{F}^{-}$

Thus, sodium and chloride ions were found to be comparatively higher in concentration and major cause of elevated levels of EC in the study area.

\section{Hydrogeochemical facies}

The piper diagram carried out by Rockworks 16 software was used to identify the hydrogeochemical facies of groundwater in the three canal catchment areas of northwest Rajasthan. As shown in Fig. 3, about 91\% (site 1), 36.9 (site 2), 100 (site 3 ) of water samples fall in zone of no dominant cation type and $9 \%$ (site 1), $73.7 \%$ (site 2 ) of water samples fall in zone of $\mathrm{Na}^{+}$sodium type. For the predominate anion, it is clearly indicated that the chloride-type sample fallen in $68 \%$ (site 1), $84.2 \%$ (site 2), $84.2 \%$ (site 3 ) shows that the prominent hydrogeological facies followed by site 1 fall in mixed $\mathrm{CaNaHCO}_{3}, \mathrm{CaHCO}_{3}, \mathrm{NaCl}, \mathrm{CaMgClSO}_{4}$; site 2 water samples fall in $\mathrm{CaCl}, \mathrm{NaCl}, \mathrm{NaKClSO}_{4}$; site-3 water samples fall in $\mathrm{CaCl}, \mathrm{CaMgClSO}_{4}$ (Table 7). The diagram also shows that there are different water types present in the groundwater systems. These trilinear diagrams are beneficial in bringing out chemical relationships among groundwater samples rather than plotting methods (Piper 1944; Back and Hanshaw 1965).

\section{Irrigation suitability}

Irrigated agriculture is dependent on an adequate water supply of usable quality. Conceptually, water quality refers to the characteristics of a water supply that will influence its suitability for a specific use (Kumar et al. 2007).

The northern part of the study area is characterized by arid soils which are light yellowish brown to pale in color with calcareous concretions in the depth range of $75-100 \mathrm{~cm}$. Soils are deep and moderately drained. Permeability is moderate to moderately low, and water holding capacity and natural fertility are generally poor. Loamy sand and sandy loam are the predominant types of soil met within this soil group. At places, patches of sand also occur. The southern portion of the region is characterized by desert soils, which are very pale brown to yellowish brown in color and are generally devoid of lime concretions. Soils are generally well drained to excessively drained and have low moisture holding capacity and high permeability. So, in the poor soil conditions, irrigation water quality matters for good agriculture practices.

This section reveals the variation in the levels of the parameters and its suitability for agriculture practice (Tables 8, 9, 10).

\section{Salinity index}

Electrical conductivity is a useful and reliable index for the measurement of water salinity or total dissolved solids in water. Electrical conductivity in water is due to ionization of dissolved inorganic solids-minerals, salts, metals, cations or anions that dissolved in water. It is evident from the data in Table 11 that majority of the groundwater samples in the study were under high salinity class and suitable only for irrigating medium and high salt-tolerant crops. Salinity occurs due to weathering of rocks and release of soluble salts in the groundwater, mainly chlorides of sodium, calcium and magnesium and to a lesser extent, sulfates and carbonates. 


\section{Piper Diagram}

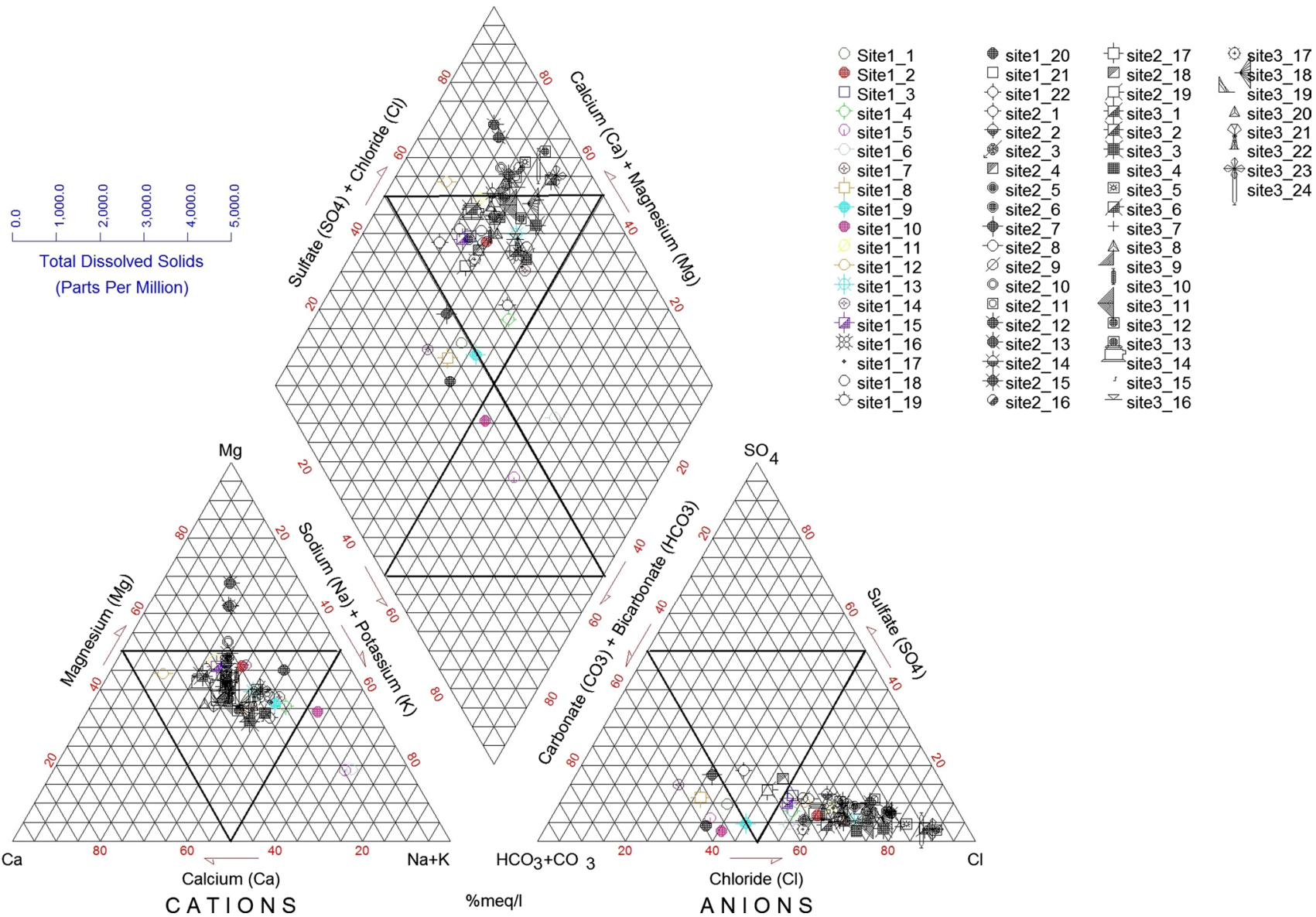

Fig. 3 Piper trilinear diagram for sites 1, 2 and 3

Table 7 Variation in hydrochemical facies for sites 1,2 and 3

\begin{tabular}{|c|c|c|c|c|c|c|c|c|}
\hline \multirow{2}{*}{\multicolumn{2}{|c|}{$\begin{array}{l}\text { Subdivision } \\
\text { of diamond }\end{array}$}} & \multirow[t]{2}{*}{ Diamond-shaped fields } & \multicolumn{2}{|l|}{ Site 1} & \multicolumn{2}{|l|}{ Site 2} & \multicolumn{2}{|l|}{ Site 3} \\
\hline & & & $\begin{array}{l}\text { No. of sam- } \\
\text { ples percent- } \\
\text { age }\end{array}$ & $\%$ & $\begin{array}{l}\text { No. of sam- } \\
\text { ples percent- } \\
\text { age }\end{array}$ & $\%$ & $\begin{array}{l}\text { No. of sam- } \\
\text { ples percent- } \\
\text { age }\end{array}$ & $\%$ \\
\hline \multirow[t]{4}{*}{ Cations } & A & Calcium type & - & - & - & - & - & - \\
\hline & $\mathrm{B}$ & No dominant & 20 & 91 & 5 & 26.3 & 24 & 100 \\
\hline & $\mathrm{C}$ & Magnesium type & - & - & - & - & - & - \\
\hline & $\mathrm{D}$ & Sodium type & 2 & 9 & 14 & 73.7 & - & - \\
\hline \multirow[t]{4}{*}{ Anions } & $\mathrm{E}$ & Bicarbonate type & 7 & 31.8 & 1 & 5.2 & - & - \\
\hline & $\mathrm{B}$ & No dominant & - & - & 2 & 10.6 & - & - \\
\hline & $\mathrm{F}$ & Sulfate type & - & - & - & - & - & - \\
\hline & $\mathrm{G}$ & Chloride type & 15 & 68.2 & 16 & 84.2 & 24 & 100 \\
\hline
\end{tabular}

Typically, irrigation water salinity in arid and semiarid areas is greater than that of humid and sub-humid areas (Bauder et al. 2007). High salinity of irrigation water is responsible for salt accumulation in the root zone and leads to damage in plant cells (Greenway and Munns 1980). Plants absorb water through a process of 'osmo-regulation,' and when the soil solution salinity is greater than the internal salinity of the plant, water uptake is restricted. High salinity can also lead to physiological drought conditions and ion toxicity (Zhu 2002). 
Table 8 Irrigation water quality parameters (mean values in $\mathrm{meq} / \mathrm{l}$ ) of groundwater samples in Indira Gandhi Canal catchment area (site 1)
Table 9 Irrigation water quality parameters (mean values in meq/l) of groundwater samples in Bhakra Canal catchment area (site 2)

\begin{tabular}{lcclllllllll}
\hline Sample ID & $\begin{array}{l}\text { No. of } \\
\text { sam- }\end{array}$ & PS & \%Na & SAR & KI & RSC & RSBC & MH & Mg/Ca & PI \\
& ples & & & & & & & & & & \\
\hline IGC1 & 5 & 3.995 & 35.41315 & 2.20 & 0.548303 & 2.80 & 3.42 & 63.70757 & 1.88 & 55.19785 \\
IGC2 & 6 & 13.6 & 28.17077 & 2.56 & 0.392191 & - & -0.09 & 65.63991 & 2.23 & 36.35815 \\
IGC3 & 4 & 8.695 & 23.44454 & 1.82 & 0.306243 & - & 0.57 & 63.60424 & 2.23 & 34.52004 \\
IGC4 & 11 & 9.22 & 43.38776 & 3.87 & 0.766402 & 3.17 & 2.07 & 64.45566 & 1.80 & 53.25211 \\
IGC5 & 7 & 5.765 & 66.10268 & 14.59 & 1.950086 & 9.63 & 7.46 & 56.28227 & 1.56 & 83.722 \\
IGC6 & 6 & 9.57 & 67.631 & 6.48 & 2.089376 & 5.33 & 4.93 & 59.52782 & 1.45 & 81.65169 \\
IGC7 & 5 & 17.475 & 40.69249 & 4.37 & 0.686127 & - & 1.68 & 65.78035 & 2.24 & 49.31494 \\
IGC8 & 2 & 3.14 & 34.3696 & 1.99 & 0.523684 & - & 3.6 & 55.26316 & 1.40 & 56.15043 \\
IGC9 & 3 & 8.25 & 40.32342 & 3.52 & 0.675699 & 3.90 & 5.4 & 62.67483 & 3.57 & 55.33263 \\
IGC10 & 3 & 4.61 & 50.28902 & 3.10 & 1.011628 & 4.17 & 5.24 & 72.28682 & 4.7 & 71.98002 \\
IGC11 & 7 & 12.8 & 21.10429 & 1.81 & 0.267496 & - & -3.62 & 61.46967 & 1.67 & 28.43122 \\
IGC12 & 4 & 7.785 & 10.99531 & 0.69 & 0.123536 & - & -3.18 & 50.64403 & 1.15 & 22.6171 \\
IGC13 & 5 & 16.59 & 34.19355 & 3.48 & 0.519608 & - & -1.72 & 61.17647 & 1.74 & 41.70111 \\
IGC14 & 2 & 1.71 & 28.50838 & 1.60 & 0.398765 & - & 3.3 & 66.66667 & 2.05 & 49.13053 \\
IGC15 & 3 & 7.835 & 22.45455 & 2.46 & 0.289566 & 5.2 & -0.4 & 60.551 & 6.63 & 33.46476 \\
IGC16 & 6 & 14.425 & 37.56801 & 3.76 & 0.601743 & 0.30 & -1.67 & 62.22222 & 1.67 & 44.24779 \\
IGC17 & 5 & 14.19 & 39.93447 & 3.54 & 0.664848 & - & 0.54 & 62.06061 & 1.68 & 48.92858 \\
IGC18 & 5 & 20.94 & 38.44394 & 4.37 & 0.624535 & - & -1.4 & 63.56877 & 1.84 & 44.6066 \\
IGC19 & 2 & 10.685 & 41.39584 & 3.16 & 0.706364 & 3.3 & 2.25 & 56.81818 & 1.80 & 54.61852 \\
IGC20 & 6 & 1.915 & 36.60714 & 1.49 & 0.577465 & 1.53 & 2.43 & 74.64789 & 4.67 & 66.34981 \\
IGC21 & 2 & 18.22 & 25.0673 & 2.38 & 0.334531 & - & -3.1 & 55.68862 & 1.25 & 33.21015 \\
IGC22 & 2 & 9.59 & 20.61069 & 1.88 & 0.259615 & - & -2.9 & 58.07692 & 1.40 & 28.95534 \\
\hline & & & & & & & & & &
\end{tabular}

\begin{tabular}{|c|c|c|c|c|c|c|c|c|c|c|}
\hline Sample ID & $\begin{array}{l}\text { No. of } \\
\text { sam- } \\
\text { ples }\end{array}$ & PS & $\% \mathrm{Na}$ & SAR & KI & RSC & RSBC & MH & $\mathrm{Mg} / \mathrm{Ca}$ & PI \\
\hline $\mathrm{BC} 1$ & 8 & 7.03 & 38.09074 & 2.87 & 0.615267 & 2.10 & 3.69 & 76.64122 & 24.89 & 49.59552 \\
\hline $\mathrm{BC} 2$ & 7 & 18.955 & 57.36531 & 6.61 & 1.345508 & 1.43 & 2.51 & 58.87509 & 1.34 & 65.65762 \\
\hline BC3 & 2 & 30.865 & 53.80887 & 9.16 & 1.164918 & 1.90 & -3.4 & 60.98361 & 1.65 & 57.77799 \\
\hline $\mathrm{BC} 4$ & 8 & 26.395 & 53.61871 & 7.40 & 1.156042 & 1.0 & -0.51 & 59.51475 & 1.43 & 59.46608 \\
\hline BC5 & 8 & 23.98 & 57.0229 & 7.59 & 1.326821 & 2.0 & 0.65 & 58.67377 & 1.36 & 63.56628 \\
\hline BC6 & 3 & 15.295 & 69.24129 & 9.57 & 2.251111 & 5.17 & 5.5 & 64.77778 & 1.87 & 78.68595 \\
\hline BC7 & 3 & 5.905 & 68.55346 & 6.45 & 2.18 & - & 6.3 & 56.90909 & 1.57 & 84.18538 \\
\hline $\mathrm{BC} 8$ & 5 & 18.035 & 38.72201 & 4.0 & 0.631907 & - & 0.82 & 60.87344 & 1.48 & 46.57247 \\
\hline BC9 & 6 & 17.575 & 41.27132 & 4.22 & 0.702746 & - & 1.41 & 55.54382 & 1.30 & 50.60087 \\
\hline BC10 & 6 & 30.425 & 57.80622 & 9.31 & 1.370017 & - & 0.55 & 68.45754 & 2.23 & 62.37444 \\
\hline BC11 & 2 & 23.82 & 51.6129 & 7.35 & 1.066667 & - & -3.6 & 58.73016 & 1.65 & 56.26541 \\
\hline BC12 & 5 & 36.93 & 41.07066 & 6.32 & 0.696948 & - & -2.26 & 76.11434 & 69.24 & 44.61131 \\
\hline BC13 & 2 & 36.25 & 53.50691 & 8.31 & 1.150857 & - & 2 & 80.95238 & 28.75 & 57.73283 \\
\hline BC14 & 4 & 20.91 & 54.53883 & 7.41 & 1.19968 & 1.30 & 1.07 & 65.67005 & 29.28 & 60.64136 \\
\hline BC15 & 7 & 27.705 & 46.94486 & 6.69 & 0.884831 & - & -2.21 & 63.04173 & 1.51 & 52.05681 \\
\hline BC16 & 2 & 33.695 & 58.02446 & 9.30 & 1.38234 & - & -2.6 & 59.8234 & 1.60 & 62.25319 \\
\hline BC17 & 15 & 20.29 & 54.66329 & 6.74 & 1.205718 & 1.67 & 1.02 & 57.05407 & 1.31 & 62.09062 \\
\hline BC18 & 4 & 9.185 & 54.9325 & 4.52 & 1.218894 & 2.25 & 2.55 & 57.37327 & 1.78 & 67.07692 \\
\hline BC19 & 3 & 12.25 & 57.37822 & 6.63 & 1.346218 & 6.0 & 4.7 & 58.23529 & 1.43 & 68.02867 \\
\hline
\end{tabular}


Table 10 Irrigation water quality parameters (mean values in $\mathrm{meq} / \mathrm{l}$ ) of groundwater samples in Gang Canal catchment area (site 3)

\begin{tabular}{lcllllllllll}
\hline Sample ID & $\begin{array}{l}\text { No. of } \\
\text { sam- }\end{array}$ & PS & \%Na & SAR & KI & RSC & RSBC & MH & Mg/Ca & PI \\
& ples & & & & & & & & & & \\
\hline GC1 & 5 & 15.735 & 20.33258 & 1.65 & 0.255218 & - & -1.7 & 55.78748 & 1.26 & 30.31819 \\
GC2 & 6 & 12.79 & 36.02564 & 2.62 & 0.563126 & - & -1.87 & 53.90782 & 1.28 & 44.93796 \\
GC3 & 8 & 27.77 & 37.56989 & 4.48 & 0.601791 & - & -7.32 & 51.25732 & 1.06 & 42.72161 \\
GC4 & 6 & 15.2 & 39.89491 & 2.97 & 0.663753 & 0.40 & -0.92 & 57.29657 & 1.42 & 48.72299 \\
GC5 & 6 & 34.86 & 26.36141 & 2.79 & 0.357984 & 1.40 & -8.23 & 58.25536 & 1.48 & 31.13005 \\
GC6 & 3 & 53.515 & 35.13059 & 4.98 & 0.541559 & - & -9.8 & 62.63245 & 1.67 & 38.56973 \\
GC7 & 3 & 42.625 & 35.05567 & 4.66 & 0.53978 & - & -7.96 & 57.59443 & 1.37 & 39.55092 \\
GC8 & 1 & 22.34 & 24.86988 & 2.70 & 0.331024 & - & -8.2 & 49.39759 & 1.0 & 31.23054 \\
GC9 & 3 & 19.365 & 30.40401 & 2.83 & 0.436864 & - & -3.87 & 53.38983 & 1.13 & 38.03166 \\
GC10 & 2 & 11.535 & 29.34502 & 1.82 & 0.415328 & - & 0.25 & 60.21898 & 1.65 & 41.16956 \\
GC11 & 2 & 31.94 & 30.79216 & 3.56 & 0.444923 & - & -5.8 & 55.07692 & 1.20 & 36.87363 \\
GC12 & 2 & 26.25 & 33.62128 & 3.84 & 0.506507 & - & -6.25 & 52.56849 & 1.20 & 39.66277 \\
GC13 & 2 & 71.255 & 30.16291 & 4.68 & 0.431904 & - & -20.55 & 54.63122 & 1.20 & 32.81706 \\
GC14 & 4 & 10.485 & 21.0975 & 1.49 & 0.267387 & - & -1.63 & 52.19446 & 1.13 & 32.76691 \\
GC15 & 4 & 21.265 & 40.21236 & 4.18 & 0.672587 & - & -3.33 & 53.87067 & 1.28 & 46.82749 \\
GC16 & 11 & 12.66 & 34.6969 & 2.41 & 0.531321 & - & -1.41 & 54.9434 & 1.28 & 44.7145 \\
GC17 & 3 & 8.52 & 28.08684 & 1.38 & 0.390566 & - & 0.9 & 58.20755 & 1.33 & 43.3379 \\
GC18 & 4 & 25.165 & 31.92967 & 3.79 & 0.469069 & - & -8.31 & 53.09311 & 1.23 & 37.19487 \\
GC19 & 12 & 32.085 & 30.34867 & 3.56 & 0.435723 & - & -9.4 & 52.22156 & 1.11 & 35.45221 \\
GC20 & 2 & 12.505 & 30.17196 & 2.04 & 0.43209 & - & -0.8 & 53.73134 & 1.35 & 42.04036 \\
GC21 & 1 & 22.54 & 27.62001 & 2.90 & 0.381597 & - & -5.7 & 49.65278 & 1.0 & 34.82156 \\
GC22 & 4 & 15.115 & 37.35537 & 3.15 & 0.596306 & - & -0.78 & 55.93668 & 1.33 & 47.1393 \\
GC23 & 2 & 64.045 & 35.9207 & 5.38 & 0.560566 & - & -11 & 63.39869 & 1.70 & 38.98354 \\
GC24 & 4 & 43.87 & 30.19481 & 3.81 & 0.432558 & - & -12.32 & 58.60465 & 1.43 & 33.56458 \\
\hline & & & & & & & & & &
\end{tabular}

Doneen (1964b) described potential salinity of irrigation water by pointing out that the suitability of water for irrigation is not only dependent on the concentration of soluble salts. It has been reported that the low-solubility salts precipitate and accumulate in the soil for successive irrigation, whereas the concentration of highly soluble salts increases the salinity of the soil (Siamak and Srikantaswamy 2009). The average potential salinity of the study area varied from 1.71 to $17.48,5.91$ to 33.70 and 8.52 to 71.26 for sites 1,2 and 3 , respectively. It has been reported that chloride is not adsorbed by soils, but it moves readily with the soil-water

Table 11 Classification of water based on EC (Handa 1969)

\begin{tabular}{lllll}
\hline EC $(\mu \mathrm{S} / \mathrm{cm})$ & Water salinity & \multicolumn{3}{l}{ Percent samples } \\
\cline { 3 - 5 } & & Site 1 & Site 2 & Site 3 \\
\hline $0-250$ & Low & 00 & 00 & 00 \\
$251-750$ & Medium & 13 & 07 & 07 \\
$751-2250$ & High & 28 & 16 & 20 \\
$2251-6000$ & Very high & 57 & 54 & 48 \\
$6001-10,000$ & Extensively high & 02 & 21 & 19 \\
$10,001-20,000$ & Extensively high & 00 & 02 & 06 \\
\hline
\end{tabular}

مدينة الملك عبدالعزيز

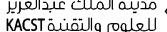

into the crop. It travels in the transpiration stream and accumulates in the leaves. Thus, excessive chloride could cause necrosis (dead tissue) in plants which is often accompanied by early leaf drop or defoliation (Ayers and Westcot 1994).

\section{Sodium percentage $(\% \mathrm{Na})$}

Sodium is one of the important metals that serve as essential nutrients necessary for some biochemical functions. It is often found in natural waters due to its high solubility and frequently associated with salinity problems when linked to chloride and sulfate ions. Percent sodium is widely utilized to assessing the suitability of water for irrigation purposes (Wilcox 1948). The \% $\mathrm{Na}$ is computed with respect to relative proportions of cations present in water and calculated using the following formula:

$\mathrm{Na} \%=\mathrm{Na}^{+} \times 100 /\left[\mathrm{Ca}^{+2}+\mathrm{Mg}^{+2}+\mathrm{Na}^{+}+\mathrm{K}^{+}\right](\mathrm{meq} / \mathrm{l})$

In this regard, the $\% \mathrm{Na}$ values and the corresponding $\mathrm{EC}$ of the waters were plotted within the Wilcox (1955) diagram (Fig. 4) to confirm the irrigation classes of the waters. Indeed, the waters were plotted within the excellent to good 


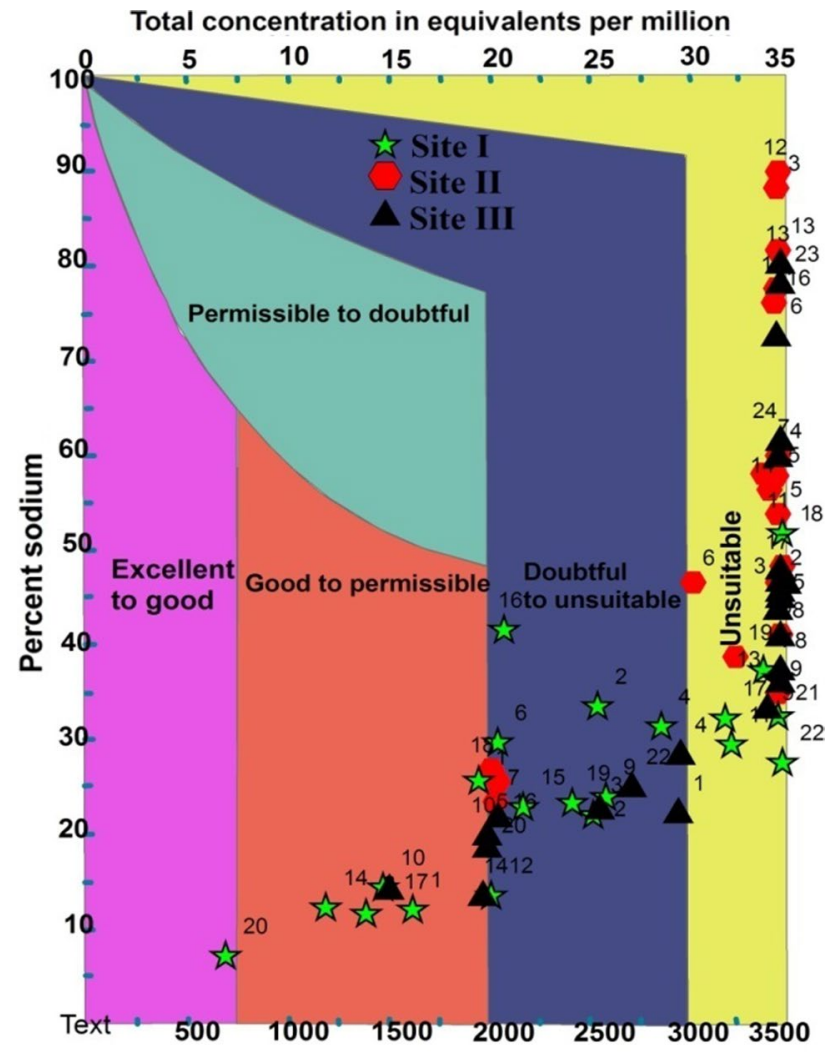

Specific conductance in micromhos per $\mathrm{cm}$ at $25 \mathrm{C}$

Fig. 4 Wilcox (1955) diagram in the study area

class, but most of the samples belong to doubtful to unsuitable class on account of the high \% Na. Considering the irrigation water quality ratings on the basis of $\% \mathrm{Na}$ (Table 12 ) reveals that majority of samples fall in good to permissible class but in combination with high electrical conductance making water unsuitable for irrigation use. Dissolution of minerals from lithological compositions and addition of chemical fertilizers are major causes of high $\mathrm{Na} \%$. Excess sodium concentration in irrigation water produces the undesirable effects. Soil dispersion is the primary physical process associated with high sodium concentration which is further assigned to reduced infiltration, reduced hydraulic

Table 12 Irrigation water quality of groundwater based on sodium percentage in study area

\begin{tabular}{lllll}
\hline Sodium (\%) & Water class & \multicolumn{3}{l}{ Percent samples } \\
\cline { 3 - 5 } & & Site 1 & Site 2 & Site 3 \\
\hline$<20$ & Excellent & 17 & - & 14 \\
$20-40$ & Good & 46 & 21 & 73 \\
$40-60$ & Permissible & 22 & 51 & 13 \\
$60-80$ & Doubtful & 13 & 28 & - \\
$>80$ & Unsuitable & 02 & - & - \\
\hline
\end{tabular}

condition and surface crusting (Shainberg and Letey 1984; Hanson et al. 1999; Bardhan et al. 2007). Increasing sodium concentration disperses soil and increasing salinity flocculates soil (Hanson et al., 1999). High $\mathrm{Na}^{+}$interferes with $\mathrm{K}^{+}$ and $\mathrm{Ca}^{+2}$ nutrition in plants and disturbs efficient stomatal opening and results in depression of photosynthesis and growth (Tayakkoli et al. 2010).

\section{Sodium adsorption ratio (SAR)}

SAR is an important parameter for the determination of suitability of water for irrigation purpose because it is responsible for the sodium hazard in irrigation water. The proportion of sodium $\left(\mathrm{Na}^{+}\right)$to calcium $\left(\mathrm{Ca}^{+2}\right)$ and magnesium $\left(\mathrm{Mg}^{+2}\right)$ ions in a water sample is expressed as SAR:

$\mathrm{SAR}=\mathrm{Na}^{+} /\left[\left(\mathrm{Ca}^{+2}+\mathrm{Mg}^{+2}\right) / 2\right]^{0.5}(\mathrm{meq} / \mathrm{l})$

The degree to which irrigation water tends to enter into cation exchange reaction in soil can be indicated by the SAR. Excess sodium or high SAR values in waters produce undesirable effects of changing soil properties and reducing soil. Irrigation waters with high SAR tend to cause soils to disperse, form crusts, become compacted and have very low infiltration rate and poor air movement properties. Irrigation waters with high SAR replace adsorbed calcium and magnesium ions with sodium ions on soil particles, and soils tend to become deflocculated and relatively impermeable. Salts present in soil solution move downward while sodium adsorbed on the soil exchange complex does not and may have a different effect on soil physical and chemical properties. A severe reduction in infiltration is likely to occur with the condition of relatively low EC and high SAR. The classification of groundwater samples based on SAR values is shown in Table 13. Majority of samples were found within the range of excellent to good category and for suitable irrigation use (except $2 \%$ samples in site 1 ).

SAR and salinity combination could be used to assess irrigation water quality in relation to infiltration or permeability problem. The US salinity diagram (Fig. 5) illustrates that most of the groundwater samples fall in the field of $\mathrm{C}_{4} \mathrm{~S}_{1}$, indicating very high salinity and low alkalinity hazard and suitable only for crops having good salt-tolerance

Table 13 Classification of irrigation water on SAR values

\begin{tabular}{lllll}
\hline SAR values & Water quality & \multicolumn{4}{l}{ Percent samples } \\
\cline { 3 - 5 } & & Site 1 & Site 2 & Site 3 \\
\hline$<10$ & Excellent & 96 & 84 & 100 \\
$10-18$ & Good & 02 & 16 & - \\
$19-26$ & Doubtful & - & - & - \\
$>26$ & Unsuitable & 02 & - & - \\
\hline
\end{tabular}




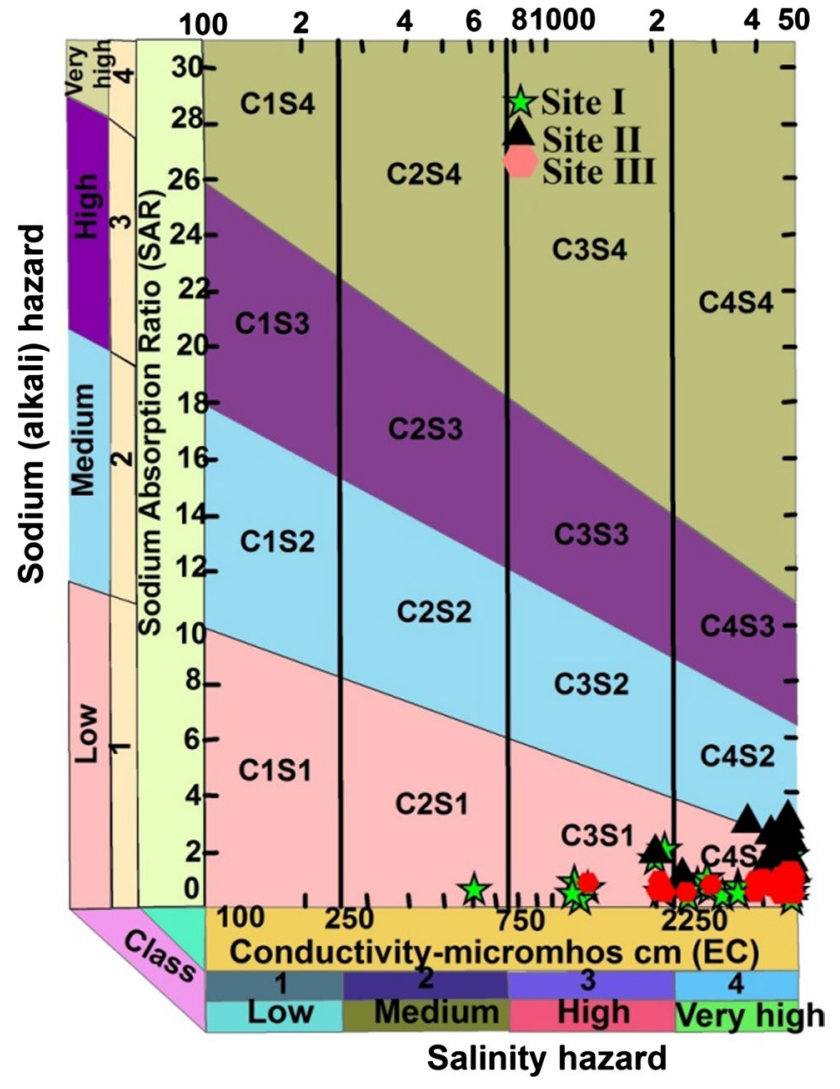

Fig. 5 USSL classification in the study area

capacity. Few samples fall in the field of $\mathrm{C}_{3} \mathrm{~S}_{1}$, indicating high salinity with low sodium content and suitability for irrigation.

\section{Kelly's index (KI)}

Sodium measured against calcium and magnesium is used to calculate KI value (Kelly 1940). However, nowadays, SAR is a more popular parameter to highlight sodium hazard and this parameter is not in common use. KI more than 1 indicates high sodium content and unsuitability for irrigation use, while the samples having KI less than 1 are suitable for irrigation. In the present study, KI values were observed in the ranges $0.12-2.09,0.62-2.25$ and $0.26-0.67 \mathrm{meq} / \mathrm{l}$ for sites 1,2 and 3 , respectively.

\section{Residual sodium carbonate (RSC)}

The excess sum of carbonate and bicarbonate in groundwater over the sum of calcium and magnesium also influences the quality of water. High carbonate and bicarbonate in water essentially increase the sodium hazard of the water to a level greater than that indicated by the SAR. High carbonate and bicarbonate tend to precipitate calcium carbonate and
Table 14 Groundwater quality based on RSC (Richards 1954)

\begin{tabular}{lllll}
\hline RSC (meq/l) & Water quality & \multicolumn{4}{l}{ Percent samples } \\
\cline { 3 - 5 } & & Site 1 & Site 2 & Site 3 \\
\hline$<1.25$ & Good & 81 & 84 & 99 \\
$1.25-2.50$ & Doubtful & 03 & 08 & 01 \\
$>2.50$ & Unsuitable & 16 & 08 & - \\
\hline
\end{tabular}

magnesium carbonate when the soil solution is concentrated during soil drying.

RSC is an important parameter to evaluate the suitability of irrigation water and calculated by the following formula:

$\mathrm{RSC}=\left(\mathrm{HCO}_{3}^{-}+\mathrm{CO}_{3}^{-2}\right)-\left(\mathrm{Ca}^{+2}+\mathrm{Mg}^{+2}\right)(\mathrm{meq} / \mathrm{l})$

The classification of irrigation water based on RSC values (Table 14) represents that most of the samples belong to good category.

Gupta and Gupta (1987) postulated residual sodium bicarbonate (RSBC) parameter for groundwater suitability and suggested the value $<5 \mathrm{mg} / \mathrm{L}$ to be most appropriate. It is calculated by the following formula:

$\mathrm{RSBC}=\mathrm{HCO}_{3}^{-}-\mathrm{Ca}^{+2}(\mathrm{meq} / \mathrm{l})$

In the study area, RSBC varied between -3.62 and 7.46, -3.6 and 6.3 and -20.55 and $0.9 \mathrm{meq} / \mathrm{l}$ for the sites 1,2 and 3, respectively. Calcium is one of the most abundant natural elements in the environment and generally found in all natural waters. It has higher concentration in water from limestone area when compared to that from non-calcareous area.

\section{Magnesium hazard (MH)}

Magnesium content of water is considered as one of the most important qualitative criteria in determining the quality of water for irrigation. A high level of magnesium is usually due to the presence of exchangeable sodium in irrigated soils. MH was introduced by Paliwal (1972) and calculated as

$\mathrm{MH}=\mathrm{Mg}^{+2} \times 100 /\left(\mathrm{Ca}^{+2}+\mathrm{Mg}^{+2}\right)(\mathrm{meq} / \mathrm{l})$

MH value more than $50 \%$ has an adverse affects on the crop yield and soil structure. In the present study area, all the water samples had values more than $50 \%$, which were unsuitable for irrigation purpose. Generally, calcium and magnesium maintain a state of equilibrium in most waters. More magnesium in water will adversely affect crop yields as the soils become more alkaline. Considering the irrigation water quality ratings based on $\mathrm{Mg} / \mathrm{Ca}$ ratio shows that 
Table 15 Classification of irrigation water based on $\mathrm{Mg} / \mathrm{Ca}$ ratio

\begin{tabular}{lllll}
\hline $\mathrm{Mg} / \mathrm{Ca}$ ratio & Water quality & \multicolumn{3}{l}{ Percent samples } \\
\cline { 3 - 5 } & & Site 1 & Site 2 & Site 3 \\
\hline$<1.5$ & Safe & 43 & 57 & 78 \\
$1.5-3.0$ & Moderate & 45 & 35 & 22 \\
$>3.0$ & Unsafe & 12 & 08 & - \\
\hline
\end{tabular}

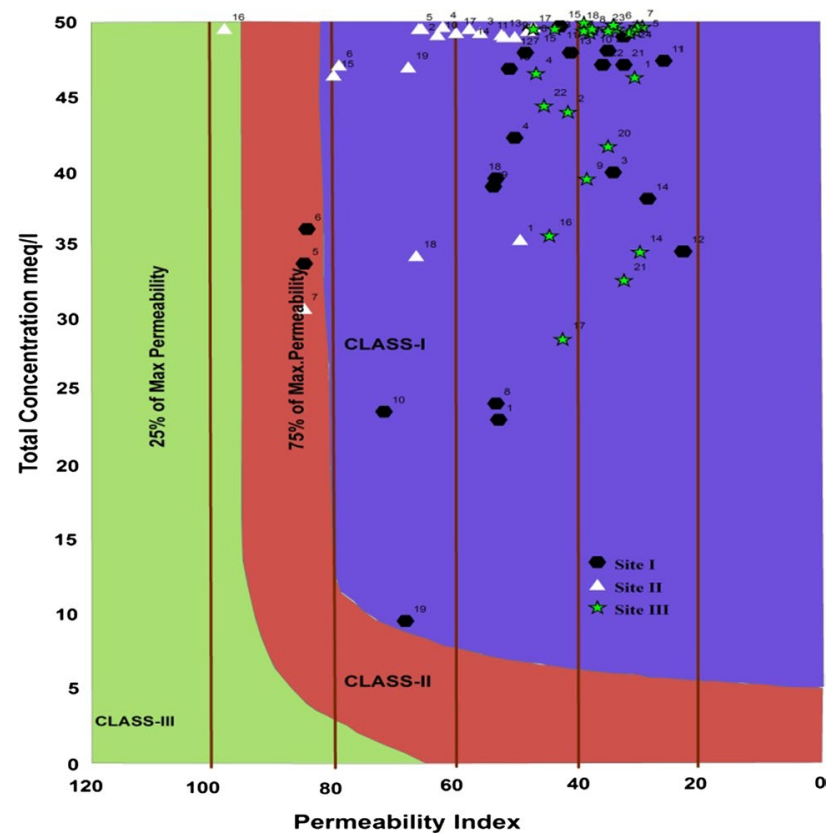

Fig. 6 Doneen classification of irrigation water based on permeability index $(\mathrm{PI})$

majority of the samples belong to safe and moderate category (Table 15).

\section{Permeability index (PI)}

As an extension, Doneen's (1964a) model for assessing the suitability of water for irrigation based on the PI was also used to classify the waters. PI is calculated by the following equation:

$\mathrm{PI}=\left[\mathrm{Na}^{+}+\left(\mathrm{HCO}_{3}^{-}\right)^{0.5}\right] \times 100 /\left[\mathrm{Na}^{+}+\mathrm{Ca}^{+2}+\mathrm{Mg}^{+2}\right]$

Accordingly, waters can be classified as Class I, Class II and Class III orders. Class I and Class II waters are categorized as good for irrigation with $75 \%$ PI or more. Class III water is unsuitable with $25 \%$ of maximum permeability. The calculated PI value ranges from 22.62 to $83.72,44.61$ to 84.19 and 30.32 to 48.72 in sites 1,2 and 3, respectively (Fig. 6).

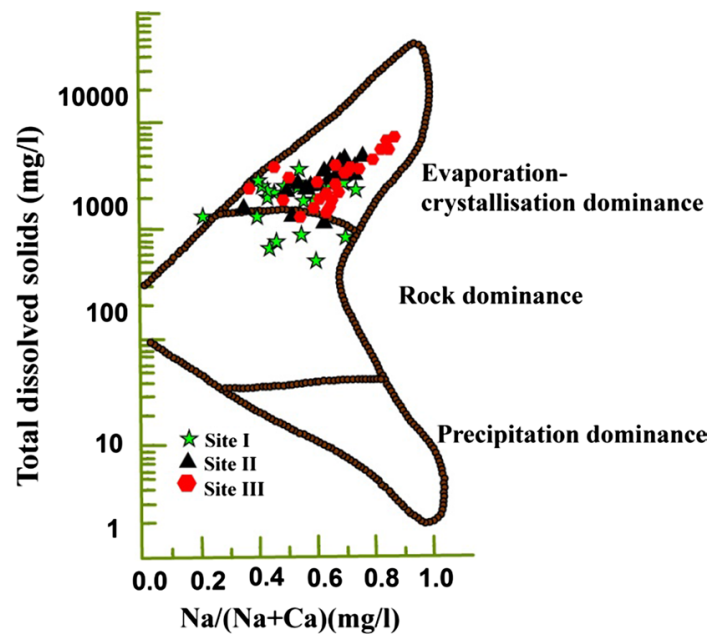

Fig. 7 Gibbs variation diagram (TDS vs. [(Na+K)/(Na+K+Ca)]

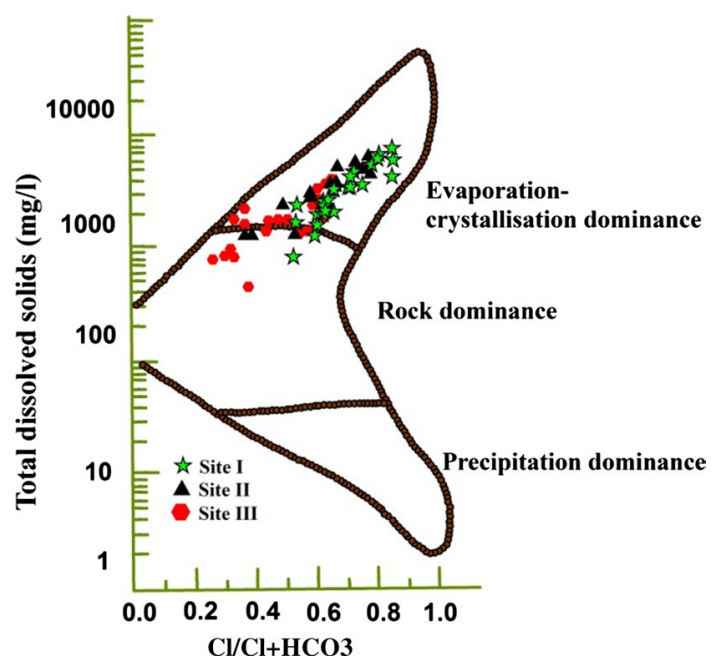

Fig. 8 Gibbs variation diagram (TDS vs. $\left[\mathrm{Cl} /\left(\mathrm{Cl}+\mathrm{HCO}_{3}\right)\right]$

\section{Gibbs groundwater chemistry}

Gibbs diagram (Gibbs 1970) represents the ratio 1 for cations $[(\mathrm{Na}+\mathrm{K}) /(\mathrm{Na}+\mathrm{K}+\mathrm{Ca})]$ and ratio 2 for anions $[\mathrm{Cl} /$ $\left.\left(\mathrm{Cl}+\mathrm{HCO}_{3}\right)\right]$ as a function of TDS to assess the groundwater chemistry. Figures 7 and 8 show that majority of the water samples belong to evaporation-crystallization zone which increases salinity by increasing $\mathrm{Na}^{+}$and $\mathrm{Cl}^{-}$with relation to increase of TDS. However, a few samples indicate the dissolution of rock minerals influencing groundwater chemical composition. 


\section{Conclusions}

The objective of this paper is to help the reader better understanding the geochemistry of groundwater in three different canal catchment areas of northwest Rajasthan. It was found that geology itself is the main cause of deteriorated groundwater quality in this area. High levels of TDS and $\mathrm{F}^{-}$are major water quality issues in drinking purpose, and community-based quality control equipments should be installed to avoid human health issues. Wise use of nitrogen fertilizers will reduce the risk of nitrate enrichment in groundwater. Mixing of canal water with groundwater could be the safe alternative in both drinking and irrigation. Selection of salttolerance species and frequent irrigation might reduce the risk of agricultural damage in the study area.

Open Access This article is distributed under the terms of the Creative Commons Attribution 4.0 International License (http://creativeco mmons.org/licenses/by/4.0/), which permits unrestricted use, distribution, and reproduction in any medium, provided you give appropriate credit to the original author(s) and the source, provide a link to the Creative Commons license, and indicate if changes were made.

\section{References}

APHA (1989) Standard methods for examination of water and wastewater, 17th edn. American Public Health Association, Washington, DC

APHA (1995) Standard methods for the examination of water and wastewater, 19th edn. American Public Health Association, Washington, DC

Arumugam K, Elangovan K (2009) Hydrochemical characteristics and groundwater quality assessment in Tirupur Region Coimbatore District, Tamil Nadu, India. Environ Geol 58:1509-1520

Ayers RS, Westcot DW (1994) Water quality for agriculture. FAO Irrig Drain Pap 29(1):1-130

Back W, Hanshaw BB (1965) Chemical geohydrology In: Advances in hydroscience, Academic Press, New York, pp 11-49

Bardhan G, Chaudhari SK, Mohapatra PK (2007) Effect of irrigation water quality on saturated hydraulic conductivity of typic Haplustert, Vertic Haplustept, and Lithic Ustorthent Soils. J Agric Phys 7:38-46

Bauder TA, Cardon GE, Waskam RM, Davis JG (2004) Irrigation water quality-cooperative extension agriculture. Colorado State University, Colorado

Bauder TA, Waskom RM, Davis JG (2007) Irrigation water quality criteria, US Department of Agriculture, Colorado State University, Colorado

Bureau of Indian Standards (2003) Drinking water-specification IS: 10500. BIS, New Delhi

Chaudhary V, Sharma M, Yadav BS (2008) Assessment of water fluoride toxicity levels in northwest Rajasthan, India. Fluoride 41(3):212-215

Chaudhary V, Sharma M, Yadav BS (2009) Elevated fluoride in canal catchment soils of northwest Rajasthan, India. Fluoride 42(1):46-49
Chaudhary V, Kumar M, Sharma M, Yadav BS (2010) Fluoride, boron and nitrate toxicity in groundwater of northwest Rajasthan, India. Environ Monit Assess 161:343-348

Davis SN, DeWiest RJ (1966) Hydrogeology. Wiley, New York

Doneen LD (1964a) Notes on water quality in agriculture. Published as a water science and engineering paper, 4001, Department of Water Science and Engineering, University of California

Doneen LD (1964b) Notes on water quality in agriculture. Water Science and Engineering, University of California, Davis

Drever JI (1988) The geochemistry of natural water Englewood Cliffs, vol 437. Prentice Hall, New Jersey

Fianko JR, Nartey VK, Donkor A (2010) The hydrochemistry of groundwater in rural communities within the Tema Disrict, Ghana. Environ Monit Assess 168:441-449

Garg VK, Suthar S, Singh S, Sheoran A (2009) Drinking water quality in villages of southwestern Haryana, India: assessing human health risks associated with hydrochemistry. Environ Geol 58:1329-1340

Gibbs RJ (1970) Mechanisms controlling world water chemistry. Science 170(3962):1088-1090

Gleeson T, Yoshihidewada BM, Beek LPH (2012) Water balance of global aquifers revealed by groundwater footprint. Nature 488:197-200

Greenway H, Munns R (1980) Mechanism of salt tolerance in non halophytes. Ann Rev Plant Physiol 3(1):149-190

Gupta AK, Gupta IC (1987) Management of saline soils and waters. Oxford and IBH Publishing Company, New Delhi

Handa BK (1969) Description and classification of media for hydrogeochemical investigations. In: Symposium on ground water studies in arid and semiarid regions, Roorkee

Hanson B, Grattan SR, Fulton A (1999) Agricultural salinity and drainage. University of California Irrigation Program. University of California, Davis

Hem JD (1985) Study and interpretation of the chemical characteristics of natural water. USGS, Water Supply 2254 (264):117-120 in India. Nature 460:999-1002

Jain CK, Bandyopadhyay A, Bhadra AA (2010) Assessment of groundwater quality for drinking purpose, District Nainital Uttarakhand, India. Environ Monit Assess 166:663-676

Kelly WP (1940) Permissible composition and concentration of irrigation waters. Proc ASCE 66:607

Krouse HR, Mayer B (1999) Sulfur and oxygen isotopes in sulphate. In: Herczeg AL, Cook PG (eds) Environmental tracers in subsurface hydrology. Kluwer, Dordrecht, pp 195-231

Kumar M, Kumari K, Ramanathan AL, Saxena R (2007) A comparative evaluation of groundwater suitability for irrigation and drinking purposes in two intensively cultivated districts of Punjab, India. Environ Geol 53:553-574

Kumar SK, Rammohan V, Sahayam JD, Jeevananadam M (2009) Assessment of groundwater quality and hydrogeochemistry of Manimuktha river basin, Tamil Nadu, India. Environ Monit Assess 159:341-351

Kurdi M, Tabasi S, Eslamkish T, Hezarkhani A (2013) Hydrogeochemical study to evaluate the suitability of water for irrigation purpose at Qareh sou catchment, North of Iran. Elixir

Little J, Kalischuk A, Gross D, Sheedy C (2010) Assessment of Water Quality in Alberta's Irrigation Districts. Alberta Agriculture and Rural Development, Alberta, Canada

MacDonald AM, Bonsor HC, Ahmed KM et al (2016) Groundwater quality and depletion in the Indo-Gangetic basin mapped from in situ observations. Nat Geosci. https://doi.org/10.1038/ngeo2791

Meenakshi GV, Kavita R, Malik A (2004) Groundwater quality in some villages of Haryana, India: focus on fluoride and fluorosis. J Hazard Mater 106:85-97 
Paliwal KV (1972) Irrigation with saline water. Monogram no. 2, new series 198, IARI, New Delhi

Piper AM (1944) A graphical procedure in the geochemical interpretation of water analysis. Am Geophys Union Trans 25:914-928

Rajmohan N, Elango L (2005) Nutrient chemistry of groundwater in an intensively irrigated region of Southern India. Environ Geol 47:820-830

Richards LA (1954) Diagnostics and improvement of saline and alkaline soils. U.S. Dept. of Agriculture hand book no. 60. U.S. Salinity Laboratory, Washington, DC

Rodell M, Velicogna I, Famiglietti JS (2009) Satellite-based estimates of groundwater depletion in India. Nature 460(7258):999-1002

Salifu M, Yidana SM, Osae S, Armah YS (2013) The influence of the unsaturated zone on the high fluoride contents in groundwater in the Middle Voltaian Aquifers-the Gushegu District, Northern Region of Ghana. J Hydrogeol Hydrol Eng 2:2

Sarkar AA, Hassan AA (2006) Water quality assessment of a groundwater basin in Bangladesh for irrigation use. Pak J Biol Sci 9(9):1677-1684

Sawyer GN, McMcartly DL (1967) Chemistry of sanitary engineers, 2nd edn. McGraw Hill, New York

Sengupta P (2013) Potential health impacts of hard water. Int J Prev Med 4(8):866-875

Shainberg I, Letey J (1984) Response of soils to sodic and saline conditions. Hilgardia 61:21-57

Siamak G, Srikantaswamy S (2009) Analysis of agricultural impact on the Cauvery river water around KRS dam. World Appl Sci J 6(8):1157-1169

Stallard RF, Edmond JM (1983) Geochemistry of the Amazon 2. The influence of geology and weathering environment on the dissolved load. J Geophys Res 88:9671-9688

Subba Rao N (2003) Groundwater quality: focus on fluoride concentration in rural parts of Guntur district, Andhra Pradesh, India. Hydrol Sci 48(5):835-847
Subba Rao N, Prakasa Rao J, Devadas JD, Srinivasa Rao KV, Krishna C, Nagamalleswara Rao B (2002) Hydrogeochemistry and groundwater quality in a developing urban Environment of a semi-arid region, Guntur, Andhra Pradesh, India. J Geol Soc India 59:159-166

Subramani T, Elango L, Damodarasamy SR (2005) Groundwater quality and its suitability for drinking and agricultural use Chithar River Basin, Tamil Nadu, India. Environ Geol 47:1099-1110

Tayakkoli E, Rengasamy P, McDonald E (2010) High concentrations of $\mathrm{Na}^{+}$and $\mathrm{Cl}^{-}$ions in soil solution have simultaneous detrimental effects on growth of faba bean under salinity stress. J Exp Bot 61(15):4449-4459

Todd DK (1980) Groundwater hydrology, 2nd edn. Wiley, New York

USSL (1954) Diagnosis and improvement of saline and alkali soil. USDA Hand Book no. 60, Washington

WHO (2004) Guidelines for drinking water quality: training pack. WHO, Geneva

Wilcox LV (1948) The quality of water for irrigation uses. US Dept Agri Tech Bull 962. USDA, Washington, DC

Wilcox LV (1955) Classification and use of irrigation waters. USDA, Circular 969, Washington, DC

Zhu JK (2002) Salt and drought stress signal transduction in plants. Annu Rev Plant Biol 53:247-273

Publisher's Note Springer Nature remains neutral with regard to jurisdictional claims in published maps and institutional affiliations. 